\title{
Collider and dark matter searches in the inert doublet model from Peccei-Quinn symmetry
}

\author{
Alexandre Alves, ${ }^{a}$ Daniel A. Camargo, ${ }^{b}$ Alex G. Dias, ${ }^{b}$ Robinson Longas, ${ }^{c}$ \\ Celso C. Nishi ${ }^{d}$ and Farinaldo S. Queiroz ${ }^{e}$ \\ ${ }^{a}$ Departamento de Ciências Exatas e da Terra, Universidade Federal de São Paulo, \\ Diadema-SP, 09972-270, Brasil \\ ${ }^{b}$ Universidade Federal do ABC, Centro de Ciências Naturais e Humanas, \\ 09210-580, Santo André-SP, Brasil \\ ${ }^{c}$ Instituto de Física, Universidad de Antioquia, \\ Calle 70 No. 52-21, Medellín, Colombia \\ ${ }^{d}$ Universidade Federal do ABC, Centro de Matemática, Computação e Cognição Naturais, \\ 09210-580, Santo André-SP, Brasil \\ e Max-Planck-Institut fur Kernphysik, \\ Saupfercheckweg 1, 69117 Heidelberg, Germany \\ E-mail: aalves@unifesp.br, dacamargov@gmail.com, \\ alex.dias@ufabc.edu.br, robinson. longas@udea.edu.co, \\ celso.nishi@ufabc.edu.br, farinaldo.queiroz@mpi-hd.mpg.de
}

ABstract: Weakly Interacting Massive Particles (WIMPs) and axions are arguably the most compelling dark matter candidates in the literature. Could they coexist as dark matter particles? More importantly, can they be incorporated in a well motivated framework in agreement with experimental data? In this work, we show that this two component dark matter can be realized in the Inert Doublet Model in an elegant and natural manner by virtue of the spontaneous breaking of a Peccei-Quinn $\mathrm{U}(1)_{P Q}$ symmetry into a residual $\mathbb{Z}_{2}$ symmetry. The WIMP stability is guaranteed by the $\mathbb{Z}_{2}$ symmetry and a new dark matter component, the axion, arises. There are two interesting outcomes: (i) vector-like quarks needed to implement the Peccei-Quinn symmetry in the model may act as a portal between the dark sector and the SM fields with a supersymmetry-type phenomenology at colliders; (ii) two-component Inert Doublet Model re-opens the phenomenologically interesting $100-500 \mathrm{GeV}$ mass region. We show that the model can successfully realize a two component dark matter framework and at the same time avoid low and high energy physics constraints such as monojet and dijet plus missing energy, as well as indirect and direct dark matter detection bounds.

Keywords: Beyond Standard Model, Cosmology of Theories beyond the SM, Discrete Symmetries

ARXIV EPRINT: 1606.07086 


\section{Contents}

1 Introduction 1

2 The model 3

$\begin{array}{lll}3 & \text { Collider constraints } & 7\end{array}$

3.1 Bounds from SUSY and DM searches in jets plus missing energy and monojets 7

4 Dark matter phenomenology 12

$\begin{array}{ll}4.1 & \text { WIMP relic density } \\ & 12\end{array}$

$\begin{array}{lll}4.2 & \text { Axion relic density } & 13\end{array}$

4.3 Mixed WIMP-axion dark matter in the IDM 13

$\begin{array}{ll}4.4 \text { New coannihilations with vector-like quarks } & 14\end{array}$

$\begin{array}{ll}4.5 \text { Direct detection } & 16\end{array}$

$\begin{array}{lll}4.6 & \text { Indirect detection } & 18\end{array}$

$\begin{array}{llr}5 & \text { Conclusions } & 18\end{array}$

$\begin{array}{llr}6 & \text { Prospects } & 19\end{array}$

A Simple UV completions $\quad 20$

A.1 $\mathrm{U}(1)_{\mathrm{PQ}}$ breaking in the Higgs potential 20

$\begin{array}{lll}\text { A.2 Lighter exotic quark mass } & 21\end{array}$

\section{Introduction}

The nature of dark matter (DM) is one of the most puzzling mysteries of astroparticle physics. Dark matter which accounts for $27 \%$ of the total energy density of the Universe is often interpreted in terms of WIMPs. More precisely, only one species makes up the entire DM of the universe. Although, this minimal scenario having just one particle making up the entire DM sounds appealing, there is no observational evidence supporting this idea. In fact, both matter and radiation components of the Universe energy budget is comprised of more than one particle. Thus it is rather possible that DM is constituted by more than one particle.

Albeit, having as a second WIMP merely to account for some fraction of the relic density is not so compelling, unless two solid signals are observed consistent with the two WIMPs scenario. Hence, an ideal scenario would occur if the second DM component solves a major problem in particle physics such as the Strong CP problem. This is precisely our case understudy, where the second component is the axion field. As we shall see, the addition of axions to the WIMP paradigm has two motivating outcomes: the WIMP is 
stability is naturally addressed; the strong CP problem in the Standard Model (SM) is solved. To put this framework into perspective we need to briefly review how this come into play.

WIMPs that are the most popular candidate for DM suggest a connection between DM physics and the weak scale. The stability of the WIMP is usually assumed to be due to the presence of ad hoc discrete global symmetry, such as a $\mathbb{Z}_{2}$ symmetry, which prevents its decay. Another candidate is the axion [1,2], which is the pseudo Nambu-Goldstone of the breakdown of the $\mathrm{U}(1)_{P Q}$ Peccei-Quinn (PQ) symmetry proposed to solve the strong $\mathrm{CP}$ problem [3] (see refs. [4-6] for a review). Under the assumption that the $\mathrm{U}(1)_{P Q}$ symmetry is broken at an energy scale much higher than the electroweak scale, the axion can be an ultralight particle with faint interactions with all other particles [7-10], and allowed to have a lifetime larger than the age of the Universe. The axion contribution to the total DM energy density in the Universe also depends on the energy scale in which the $\mathrm{U}(1)_{P Q}$ symmetry is broken [11].

Thus, the scenario in which both WIMP and axion make up the DM of the Universe is a natural and compelling framework. With that in mind we add a new and well motivated ingredient, the axion, in one of the simplest extensions of the Standard Model with a WIMP: the Inert Doublet Model (IDM), which contains an additional SU $(2)_{L}$ Higgs doublet with the lightest component stabilized by an ad hoc $\mathbb{Z}_{2}$ symmetry [12-14].

In other words, we propose the axion as the DM companion to the IDM component $H^{0}$. To this end we have developed a model based on the observation made in [15], where a $\mathrm{U}(1)_{P Q}$ symmetry broken spontaneously into a $\mathbb{Z}_{2}$ symmetry was advocated to stabilize the WIMP. ${ }^{1}$ We tacitly assume that the $\mathrm{U}(1)_{P Q}$ symmetry is protected against gravitational effects - which generate Planck-scale-suppressed symmetry breaking operators — by some sort of discrete symmetry (as in e.g. [23, 24]) to avoid destabilization of the solution to the strong CP problem, and also of the WIMP [25]. The use of this global symmetry to stabilize the WIMPs is safe from gravitational effects which might violate the $\mathrm{U}(1)_{P Q}[25]$, since only Planck suppressed operators of dimension six are present. To complete this two component DM system, at least a scalar singlet field hosting the axion $a$ and a vector-like quark $D$ are necessary in addition to the inert Higgs doublet whose lightest neutral component is the heavy DM [26-28]. The vector-like quark allows a simple implementation of the $\mathrm{U}(1)_{P Q}$ symmetry, as in the KSVZ axion model [7, 8], and acts as a portal connecting the SM and the dark sector. As a consequence of the residual $\mathbb{Z}_{2}$ symmetry, the heavy vector quarks decay only to new heavy scalars and SM quarks, mimicking the phenomenology of $R$-parity conserving supersymmetry (SUSY) at colliders, including the classic SUSY signal of jets plus large missing energy.

As there is currently many experimental constraints on supersymmetry from the LHC searches, we performed, prior to the study of the multi-component DM scenario of our model, an investigation of the limits from the searches of jets plus missing energy and monojets at the LHC. After that, we focused on the main goal of the paper, which is

\footnotetext{
${ }^{1}$ Other contexts where the WIMP is stabilized by an accidental symmetry that remains from the breaking of a more fundamental symmetry at a higher energy scale are given in [16-22].
} 
the study of our axion-WIMP DM scenario, pointing out the differences in relation to the typical IDM. The main finding is that, in contrast with the one-component DM in the IDM, the phenomenologically important mass interval $100 \mathrm{GeV} \leq M_{H^{0}} \leq 500 \mathrm{GeV}$ is re-opened, with the axion filling the role of the remaining DM.

Thus in summary, our work extends previous works in several key aspects;

- We add the axion to the WIMP paradigm in the scope of the Inert Doublet Model, naturally addressing the WIMP stability (section 2 ).

- We investigate the collider phenomenology of the model (section 3).

- We show that one can successfully have a two component dark matter model with a wealth of experimental constraints (section 4).

\section{The model}

The model consists on a KSVZ type axion model $[7,8]$ with an inert doublet $H_{D}$, whose the lightest neutral component is stabilized by a residual $\mathbb{Z}_{2}^{D}$ symmetry that remains unbroken from the original PQ symmetry. Therefore, we will have two candidates for DM: the ultralight axion and the WIMP-like lightest component of $H_{D}$.

The simplest way to implement the breaking $\mathrm{U}(1)_{\mathrm{PQ}} \rightarrow \mathbb{Z}_{2}^{D}$ is to break the PQ symmetry by a vev of a singlet scalar $S$ of $\mathrm{PQ}(S)=2$ while all other fields carry integer PQ charges. The fields carrying even or zero PQ charge will be even under the remaining $\mathbb{Z}_{2}^{D}$ whereas those carrying odd PQ charge will be odd under $\mathbb{Z}_{2}^{D}$, and thus belong to the dark sector. The conservation of $\mathbb{Z}_{2}^{D}$ requires that scalars with odd $\mathrm{PQ}$ charge be inert. As usual, the responsible for PQ symmetry breaking will host the axion in its phase as

$$
S=\frac{1}{\sqrt{2}}\left(f_{a}+\rho(x)\right) e^{i a(x) / f_{a}},
$$

where $a(x)$ is the axion field, and $f_{a}$ the axion decay constant that corresponds to the vev of $S$ in our case (a KSVZ type axion model [7, 8]). Nonperturbative QCD effects lead to a potential, which generates a mass to the axion as

$$
m_{a} \approx 6 \mathrm{meV} \times\left(10^{9} \mathrm{GeV} / f_{a}\right) .
$$

In this framework the axion couplings with matter and gauge bosons are suppressed by $f_{a}$ which, being much higher than the electroweak scale, makes the axion an ultralight particle with feeble couplings to all other particles. In fact, $f_{a}$ is constrained from astrophysical objects which would have their dynamics affected if axions interact too much with photons. For example, supernova SN1987A data constrains $f_{a}$ to be greater than $10^{9} \mathrm{GeV}[29,30]$. Still, an upper limit on the decay constant is obtained from the requirement that the axion relic density should not exceed the DM density, which gives $f_{a} \leq 10^{12} \mathrm{GeV}$ [31-34].

In addition to the SM fermions we assume that there is at least one heavy quark field $D \sim(\mathbf{1},-1 / 3)$, where the numbers inside the parenthesis represent the transformation properties under the electroweak gauge group factors $\mathrm{SU}(2)_{L}$ and $\mathrm{U}(1)_{Y}$; the case of charge 


\begin{tabular}{|c|c|c|c|c|}
\hline & $D_{L}$ & $D_{R}$ & $H_{D}$ & $S$ \\
\hline $\mathrm{SU}(3)_{C}$ & $\mathbf{3}$ & $\mathbf{3}$ & $\mathbf{1}$ & $\mathbf{1}$ \\
\hline $\mathrm{SU}(2)_{L}$ & $\mathbf{1}$ & $\mathbf{1}$ & $\mathbf{2}$ & $\mathbf{1}$ \\
\hline $\mathrm{U}(1)_{P Q}$ & -1 & 1 & -1 & 2 \\
\hline $\mathbb{Z}_{2}^{D}$ & - & - & - & + \\
\hline
\end{tabular}

Table 1. Quantum numbers of the fields beyond the SM.

$2 / 3$ exotic quark can be treated analogously. Such a quark field is formed by left- and righthanded fields $D_{L, R}$, having the following interaction with $S$

$$
\mathcal{L} \supset y S^{*} \bar{D}_{L} D_{R}+\text { h.c. },
$$

so that $\mathrm{PQ}\left(D_{L}\right)=-1$ and $\mathrm{PQ}\left(D_{R}\right)=1$. This results in a nonzero value for the anomaly coefficient, $c_{a g}=\mathrm{PQ}\left(D_{L}\right)-\mathrm{PQ}\left(D_{R}\right)=-2$, allowing the axion to have a coupling with the gluon field strength as required to solve the strong CP problem through the PecceiQuinn mechanism.

With the vev of $S$ a mass $M_{D}=y f_{a} / \sqrt{2}$ for the $D$ quark is generated through the interaction in eq. (2.3). We tune the Yukawa coupling $y \leq 10^{-6}$ in eq. (2.3), as $f_{a} \geq 10^{9} \mathrm{GeV}$, so that $M_{D}$ lies at the $\mathrm{TeV}$ scale. In the appendix A.2 it is shown how to ameliorate such a tuning by extending the model.

Besides the fields necessary to solve the strong CP problem, we augment the SM with an inert Higgs doublet $H_{D} \sim(\mathbf{1}, 1 / 2)$, with $\mathrm{PQ}\left(H_{D}\right)=-1$, in addition to the usual Higgs doublet $H \sim(\mathbf{1}, 1 / 2)$. In the limit of PQ symmetry conservation, the Higgs potential is effectively ${ }^{2}$

$$
V=\mu_{1}^{2} H^{\dagger} H+\mu_{2}^{2} H_{D}^{\dagger} H_{D}+\frac{\lambda_{1}}{2}\left(H^{\dagger} H\right)^{2}+\frac{\lambda_{2}}{2}\left(H_{D}^{\dagger} H_{D}\right)^{2}+\lambda_{3}\left(H^{\dagger} H\right)\left(H_{D}^{\dagger} H_{D}\right)+\lambda_{4}\left|H^{\dagger} H_{D}\right|^{2} .
$$

Exact PQ symmetry at the electroweak scale would imply degenerate $\mathrm{CP}$ odd and $\mathrm{CP}$ even scalars of the inert doublet, a feature that is problematic if the inert doublet accounts for all or most of the DM: direct detection searches for inelastic DM requires a mass splitting larger than $100 \mathrm{keV}[28,35]$. As PQ symmetry is broken at the scale $f_{a}$ we expect the additional PQ-violating but $\mathbb{Z}_{2}^{D}$ conserving term to be generated:

$$
\delta V=\frac{1}{2} \lambda_{5}\left(H^{\dagger} H_{D}\right)^{2}+\text { h.c. }
$$

The mass splitting is thus controlled by $\lambda_{5}$, which can be taken real. A simple completion that generates the term (2.5) is shown in appendix A.1. The fields beyond the SM, along with their quantum numbers, are collected in table 1 . The interaction between the dark sector and the SM will be given essentially from the Yukawa term (apart from the interaction term involving the standard Higgs boson and the inert Higgs doublet in the potential),

\footnotetext{
${ }^{2}$ We consider that the effective parameters already includes the effects of integrating out the heavy fields at the PQ scale.
} 
acting as an inert doublet portal,

$$
\mathcal{L} \supset y_{D} \overline{q_{L}} H_{D} D_{R}+\text { h.c. }
$$

where $q_{L} \sim(2,1 / 6)$, corresponds to the three families of SM doublets of quarks and $y_{D}$ is the Yukawa coupling. We will effectively consider that there is only one heavy vectorlike quark $D$ accessible to the LHC and relevant to DM coannihilations. The possible constraints coming from these processes and also from the DM direct detection will be one of our goals. Moreover, we will choose this $\mathrm{TeV}$ scale heavy quark to couple only to one family of SM quarks. This choice will suppress new flavor violating effects such as on $D^{0}-\bar{D}^{0}$. In particular, the case in which the exotic quark couples only to the first quark family follows by imposing minimal flavor violation: for three families of heavy quarks $D_{i L, R}$ with $D_{i L} \sim d_{i R}\left(D_{i L} \sim u_{i R}\right)$ and $D_{i R} \sim q_{i L}$ the spectrum for $D_{i}$ can be chosen hierarchical as the SM down (up) quarks and with same order and approximately diagonal Yukawa couplings (as studied in, e.g., refs. [36, 37], with the difference that in our case the light-heavy quark mixing is absent due to $\mathbb{Z}_{2}^{D}$ ). We obtain only one heavy quark interacting predominantly to the first family after integrating out the much heavier fields. ${ }^{3}$ The other cases are considered for phenomenological comparison.

The spectrum at the electroweak scale which we consider is an inert doublet model [12, 28] augmented by an axion and a vector-like quark $D$ interacting with the particles of the SM through eq. (2.6). The dark sector, odd by $\mathbb{Z}_{2}^{D}$, consists of the fields of the inert doublet $H_{D}$ and the vector quark $D$. We choose the lightest component of $H_{D}$ to be lighter than $D$ and then be part of the DM content along with the axion. It has to be noted that several models at the PQ scale can lead to this spectrum at low energies. A simple complete model that leads to this spectrum is shown in appendix A.1; it coincides with model I of ref. [15] but with a different spectrum at low energies.

The electroweak symmetry breaking is still performed by $\langle H\rangle=v / \sqrt{2}(0,1)^{\top}$, where $v=246 \mathrm{GeV}$, with the resulting $\mathrm{CP}$ even state from the doublet $H$, identified as the standard Higgs boson, denoted by $h$, with mass $m_{h}=125 \mathrm{GeV}$. The components of the inert doublet

$$
H_{D}=\left(H^{+}, \frac{H^{0}+i A^{0}}{\sqrt{2}}\right)^{\top},
$$

give rise to four physical states: a charged state $H^{+}$and its charge conjugate, a neutral and $\mathrm{CP}$ odd $A^{0}$, and a neutral and $\mathrm{CP}$ even $H^{0}$. Note that $H^{0}$ does not develop a vacuum expectation value in order to leave the remnant $\mathbb{Z}_{2}^{D}$ symmetry unbroken. Thus, the scalar potential gives rise to the quartic interaction $\frac{1}{2} \lambda_{L} h^{2} X^{2}$ where $X$ is the lightest between $H^{0}$ or $A^{0}$ and $\lambda_{L} \equiv \frac{1}{2}\left(\lambda_{3}+\lambda_{4}-\left|\lambda_{5}\right|\right)$, which quantifies the strength of the Higgs portal.

After the spontaneous symmetry breaking the scalars acquire the masses

$$
\begin{aligned}
M_{H^{ \pm}}^{2} & =\mu_{2}^{2}+\frac{1}{2} \lambda_{3} v^{2}, \\
M_{H^{0}}^{2} & =\mu_{2}^{2}+\frac{1}{2} \lambda_{345} v^{2}, \\
M_{A^{0}}^{2} & =\mu_{2}^{2}+\frac{1}{2} \bar{\lambda}_{345} v^{2},
\end{aligned}
$$

\footnotetext{
${ }^{3}$ In this case, the axion-photon coupling should change appropriately.
} 
where $\lambda_{345} \equiv \lambda_{3}+\lambda_{4}+\lambda_{5}$ and $\bar{\lambda}_{345} \equiv \lambda_{3}+\lambda_{4}-\lambda_{5}$. We can see that the scalar-pseudoscalar mass splitting is indeed controlled by $\lambda_{5}$ :

$$
M_{H^{0}}^{2}-M_{A^{0}}^{2}=\lambda_{5} v^{2} .
$$

In summary, the model has eight free parameters namely,

$$
\left\{M_{H^{ \pm}}, M_{H^{0}}, M_{A^{0}}, M_{D}, y_{D}, \lambda_{2}, \lambda_{L}, f_{a}\right\},
$$

where the first four elements in this set are the masses of the particles which are odd under $\mathbb{Z}_{2}^{D}$, with $\lambda_{5}<0$ guaranteeing $H^{0}$ to be the lightest scalar of the dark sector besides the axion. The case in which $A^{0}$ is the lightest $\mathrm{CP}$ odd scalar is directly obtained replacing $\lambda_{5} \rightarrow-\lambda_{5}$. As we describe in what follows, these parameters will be subjected to a multitude of constraints from the electroweak nature of the model which will reduce the viable parameter space considerably. These include theoretical constraints as well as various phenomenological ones.

Vacuum stability and perturbativity. Considerations such as vacuum stability and perturbativity restrict the range of parameters in (2.10). For the potential to be bounded from below, we need [26, 38]

$$
\lambda_{1} \geq 0, \quad \lambda_{2} \geq 0, \quad \lambda_{3}+\sqrt{\lambda_{1} \lambda_{2}}>0, \quad 2 \lambda_{L}+\sqrt{\lambda_{1} \lambda_{2}}>0 .
$$

To ensure the inert minimum $\left(\langle H\rangle=v / \sqrt{2}(0,1)^{\top},\left\langle H_{D}\right\rangle=(0,0)^{\top}\right)$ to be the global minimum we require [39]

$$
(\text { scalar masses })^{2} \geq 0, \quad \frac{\mu_{1}^{2}}{\sqrt{\lambda_{1}}}<\frac{\mu_{2}^{2}}{\sqrt{\lambda_{2}}} .
$$

In special, the positivity of the usual Higgs mass squared requires $\mu_{1}^{2}<0$. When oneloop effects are considered [40], this condition may not be strict [41]. We also require perturbativity of the scalar quartic couplings, assuming [40]

$$
\mid \text { quartic self-couplings }|<4 \pi, \quad| X^{\dagger} X h h \text { coupling } \mid<4 \pi \text {. }
$$

Applied to the $\left(H^{0}\right)^{4}$ coupling, the first requirement ${ }^{4}$ in (2.13) translates into $\lambda_{2} \leq \frac{4}{3} \pi \approx$ 4.19 [40]. A related constraint would be the unitarity in the scalar-scalar scattering matrix [42]. We do not impose the latter explicitly and argue that perturbativity already cuts off most of the non-unitary cases.

Electroweak bound. The first basic constraint comes from the electroweak nature of $H_{D}$ and requires that the SM gauge bosons cannot decay into the dark scalars, i.e.,

$$
M_{H^{0}}+M_{A^{0}}>m_{Z}, \quad M_{H^{0}}+M_{H^{ \pm}}, M_{A^{0}}+M_{H^{ \pm}}>m_{W} .
$$

LEP Limit. Susy searches at LEP [43] further exclude $M_{H^{0}}<80 \mathrm{GeV}$ and $M_{A^{0}}<$ $100 \mathrm{GeV}$, for $M_{A^{0}}-M_{H^{0}}>8 \mathrm{GeV}$, for the neutral scalars and $M_{H^{ \pm}}<70 \mathrm{GeV}$ for the charged one.

\footnotetext{
${ }^{4}$ Within the IDM, the second requirement in (2.13) leads to an upper bound for scalar masses of tenths of $\mathrm{TeV}$ if the correct relic abundance for $H^{0}$ is required [35].
} 
LHC - Higgs invisible width. Additionally, when $M_{H^{0}}<m_{h} / 2$, invisible Higgs decays put strong constraints on the Higgs portal coupling,

$$
\left|\lambda_{L}\right| \lesssim 0.012(0.007)
$$

for $M_{H^{0}}=60 \mathrm{GeV}\left(M_{H^{0}}=10 \mathrm{GeV}\right)$ when only $h \rightarrow H^{0} H^{0}$ is open [44]. Thus we choose hereafter

$$
M_{H^{ \pm}}, M_{A^{0}}>100 \mathrm{GeV}, M_{H^{0}}>60 \mathrm{GeV} .
$$

LHC - dilepton + missing energy data. Using dilepton plus missing energy data from the LHC, bounds have been placed in the IDM for $M_{H^{0}}<M_{W}$ (the $W$ boson mass), based on production channels such as $q \bar{q} \rightarrow Z \rightarrow A^{0} H^{0} \rightarrow Z^{\star} H^{0} H^{0} \rightarrow l^{+} l^{-} H^{0} H^{0}$. In [45] the authors were able to rule out $H^{0}$ masses below $35 \mathrm{GeV}$ at $95 \%$ C.L. with Run I data. Thus far, the Higgs resonance region, where the relic density, direct, and indirect detection bounds are satisfied is left untouched. Anyway, this mass region lies outside our scenario in (2.16). (See [46] for an old study of dilepton data in the IDM).

We have reviewed the key aspects of the model as well as existing constraints for the IDM. Hereunder we discuss collider constraints based on monojet and dijet plus missing energy data from LHC at $7-8 \mathrm{TeV}$.

\section{Collider constraints}

By virtue of the $\mathbb{Z}_{2}^{D}$ symmetry, the vector-like quarks can only decay into a quark and a new heavy scalar, including the DM $H^{0}$. Pair production of these new heavy quarks gives rise to SUSY-like signatures at colliders as jets plus missing energy, while associated production of a heavy quark and $H^{0}$ leads to monojets. Therefore, constraints from collider searches for supersymmetry and DM have to be taken into account prior to a dedicated study of our DM candidate. Let us discuss how we checked these collider bounds.

\subsection{Bounds from SUSY and DM searches in jets plus missing energy and monojets}

As aforementioned, due to the $\mathbb{Z}_{2}^{D}$ symmetry, the vector-like quark $D$ can always be pair produced $(D \bar{D}, D D, \overline{D D})$ via quark or gluon fusion, or in association with a new scalar $\left(H^{0}, A^{0}, H^{ \pm}\right)$as shown in figure 1 . In particular, in figure 1 we display representative contributions for pair production, diagrams (a)-(f), and single production in association with $H^{0}$, diagram (g).

Singlet vector-quarks $D$ can interact with the down, strange and bottom quarks via Yukawa couplings to the scalars of the model. These Yukawa couplings might be constrained by flavor physics and searches for new physics in colliders. For example, low energy physics impose constraints on the Yukawa couplings for the case where $D$ couples with more than one family of SM quarks. We thus adopt safe benchmarks to render the model free from constraints on quark flavor violation allowing $D$ to interact just with one family of SM quarks at a time through the Yukawa coupling $y_{D}$. 


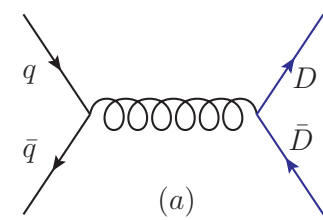

(a)

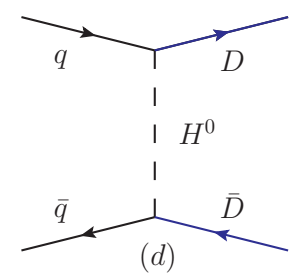

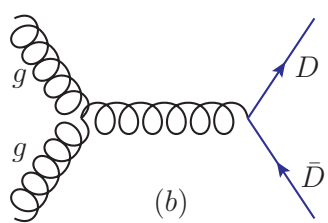

(b)
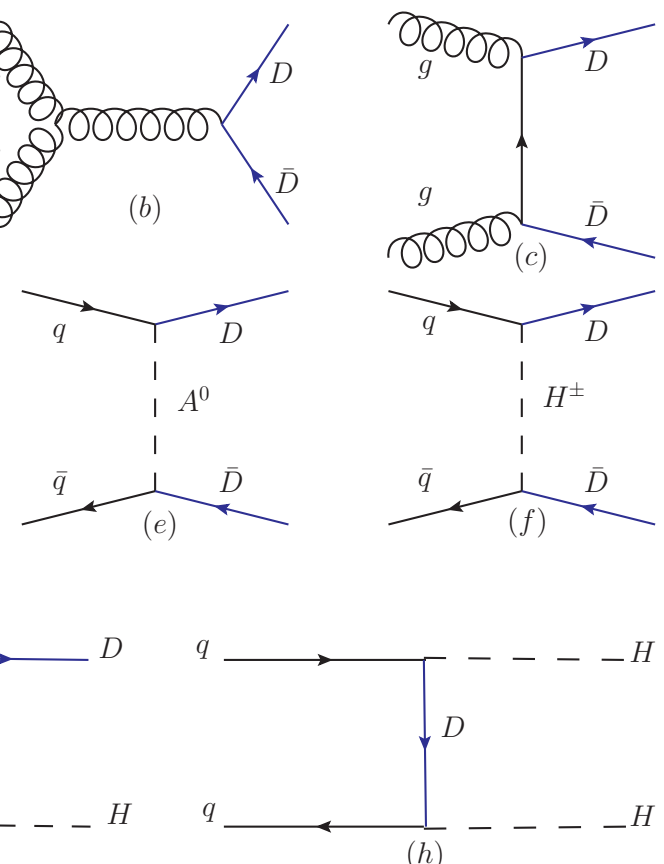

Figure 1. Feynman diagrams for production of $D \bar{D}$ pairs in proton-proton collisions are shown in diagrams (a)-(f). Additional diagrams obtained from crossing or charge conjugation of the initial and final state are not shown. In diagram (g) we display one contribution to $D+H^{0}$ associated production. Diagram (h) represents a subleading contribution to monojet signatures when a QCD jet from a strongly interacting line is radiated.

For the pair production of $D$, both QCD and Yukawa interactions with the scalars $H^{ \pm}, A^{0}, H^{0}$ contribute to the cross section. The $t$-channel diagrams with neutral scalars allow for $D D$ and $\overline{D D}$ production alongside $D \bar{D}$; see diagrams (d) and (e) in figure 1. A similar situation occurs in squark pair production where $t$-channel gluinos contribute to same-sign squarks production. Also, as in the case of squarks, the $t$-channel contributions impact significantly the production cross section of jets and missing energy.

It is shown in figure 2 the pair production cross section $\sigma\left(p p \rightarrow D_{1} D_{2}\right)$ for the $8 \mathrm{TeV}$ LHC for couplings with the first (down) and third (bottom) quark families, where $D_{1(2)}$ represents both a heavy quark and a heavy antiquark. The solid red (black) line represents the total cross section with all contributions from QCD and Yukawa couplings setting $y_{D}=1(0.5), M_{H^{0}}=400 \mathrm{GeV}$ and $M_{A^{0}}=M_{H^{ \pm}}=405 \mathrm{GeV}$. The pure QCD contribution is shown as a dashed blue line. Interestingly, the interference between the QCD and the $t$-channel Yukawa contributions is destructive, contrary to the SUSY case. The interference is visible only for the case of couplings with the first family, as shown in figure 2 where we can see at the left (right) panel the production cross section of vector-quark pairs with $d(b)-D-H^{0}$ coupling only. This is, of course, due to the parton content of the proton; the non-QCD $t$-channel diagrams connect only the initial state quarks participating in the Yukawa coupling to the vector quark $D$, thus, scenarios with exclusive couplings to the second and third families are suppressed and the Yukawa amplitudes contribute too little. 

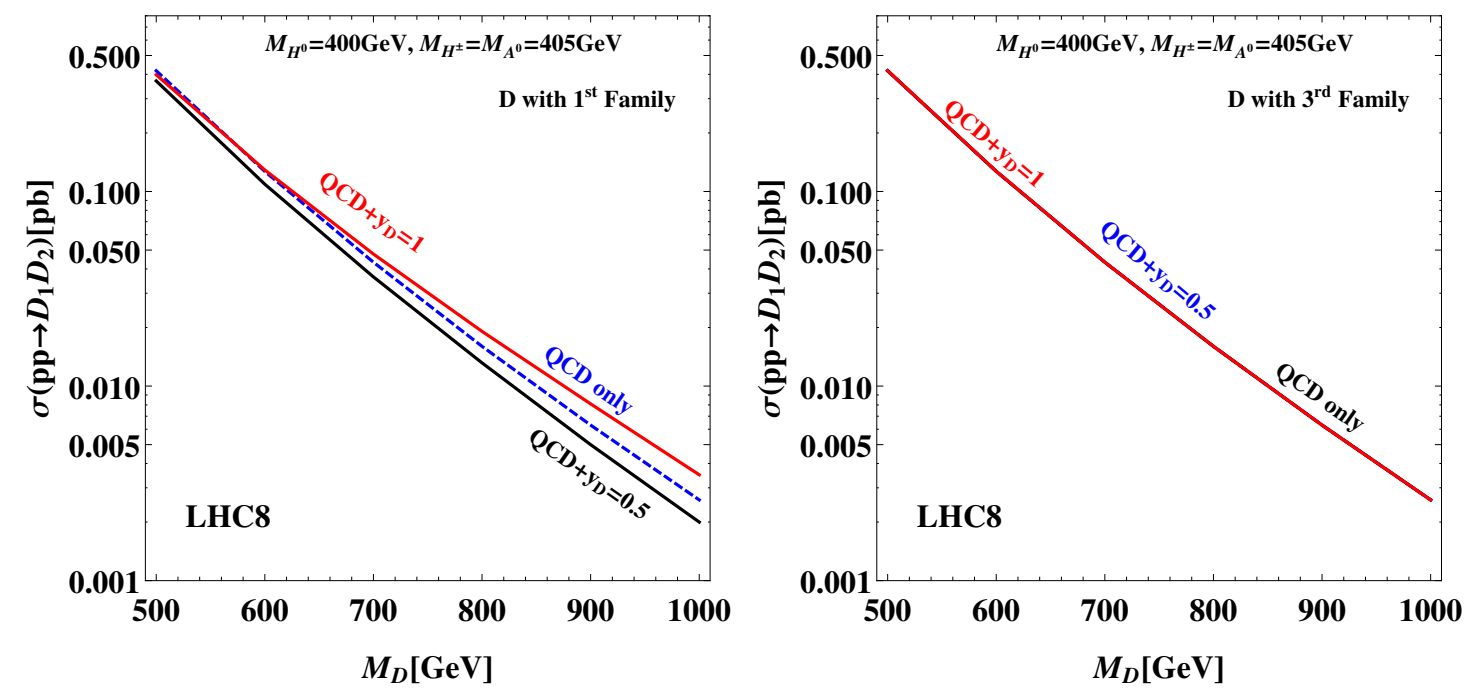

Figure 2. Pair production cross section of $D$ quark at the $\sqrt{s}=8 \mathrm{TeV}$ LHC for $D$ coupling with the first (left panel), and third (right panel) SM-generations. The result for the second family is identical to the third. The sum of the cross sections for the production of opposite-charge $(D \bar{D})$ and same-charge quarks $(D D+\overline{D D})$ as a function of the vector quark mass are displayed in solid lines. The red (black) solid line assumes $y_{D}=1(0.5)$. The blue dashed line is the QCD contribution for $D \bar{D}$ production. The scalars masses are fixed as $M_{H^{0}}=400 \mathrm{GeV}$ and $M_{A^{0}}=M_{H^{ \pm}}=405 \mathrm{GeV}$.

For moderate Yukawa couplings $y_{D} \lesssim 0.5$, the destructive interference decreases the total cross section and only at larger Yukawa coupling regimes, where $y_{D} \geq 1$, the production rate can become larger than the pure QCD contribution.

The single and pair production of the quark $D$ lead to monojet and two jets plus missing energy signatures at the LHC, respectively. Monojets also receive contributions from diagram (h) of figure 1 when a QCD jet is radiated from a strongly interacting particle. Monojets are striking signatures expected in the case that DM is produced in proton-proton collisions while two or more jets plus missing energy is the classical signature for production and decay of squarks and gluinos. Upper limits for production cross section times branching ratios for processes with hard jets and missing energy have been placed by the ATLAS and CMS Collaborations in the 7 and $8 \mathrm{TeV}$ run of the LHC, and incorporated to the database of packages aimed to check for collider limits as Smodels [47] and CheckMate [48].

As the quark $D$ has a decay channel into a jet and $H^{0}$, both the constraints from squark searches and DM searches apply in our case. In order to check these bounds we simulated the collision processes

$$
\begin{aligned}
& p p \rightarrow D \bar{D}(D D)(\overline{D D}) \rightarrow j j+E_{T} \\
& p p \rightarrow D(\bar{D}) H^{0} \rightarrow j+E_{T} \\
& p p \rightarrow H^{0} H^{0}+j \rightarrow j+E_{T}
\end{aligned}
$$

up to one extra jet to approximate higher order QCD corrections, for the $8 \mathrm{TeV} \mathrm{LHC}$, with MadGraph5 [49], Pythia6 [50] and Delphes3 [51] after implementing the model in 
FeynRules [52]. Jets are clustered with the shower- $k_{T}$ algorithm and jet matching is performed in the MLM scheme [53] at the scale $\frac{M_{D}}{4}$. We checked that differential jet rate distributions are smooth across the soft-hard jet threshold.

The processes of eq. (3.1) contribute to signatures with at least two hard jets and missing energy which mimic the production and decay of squarks and gluinos. Monojet signatures receive their main contributions from the process of eq. (3.2), with a subleading contribution from eq. (3.3) where the harder jet of the event is an initial state radiation QCD jet. Experimental searches for dark matter in monojet signatures are based on exclusive criteria to select events, discarding those events with two or more harder jets [54]. For this reason, processes like eq. (3.1), with at least two hard jets, contribute little to monojets.

Collider searches constrain the parameters related to the production cross section of the process discussed above. We have chosen to constrain the Yukawa coupling $y_{D}$ and the vector-like quark mass $M_{D}$, after fixing all the other parameters of the model. We performed scans over a wide portion of the parameters space comprising $M_{D}, M_{H^{0}}, M_{A^{0}}, M_{H^{ \pm}}$ and $y_{D}$. For each of these points we generated $10^{4}$ events for further analysis. The parameters scans were made as follows:

- First, with $M_{D}$ fixed, we varied $M_{H^{0}}, M_{A^{0}}, M_{H^{ \pm}}$and $y_{D}$ starting with $M_{H^{0}}=$ $100 \mathrm{GeV}, M_{A^{0}}=M_{H^{ \pm}}=105 \mathrm{GeV}$ until reaching almost the degeneracy of $D$ and the scalars, always keeping the hierarchy $M_{D}>\left(M_{H^{0}}=M_{A^{0}, H^{ \pm}}-5 \mathrm{GeV}\right)$, and varying the Yukawa couplings in the range $0.01 \leq y_{D} \leq 1$;

- Second, we varied $M_{D}$ in steps of $100 \mathrm{GeV}$ starting with $M_{D}=300 \mathrm{GeV}$ up to $M_{D}=1.2 \mathrm{TeV}$, proceeding with the first step for each $D$ mass.

We used SmodelS [47] to check for SUSY bounds and CheckMate [48] for monojet bounds. While the main input for SModels is the full model definition given by the SLHA file containing masses, branching ratios and cross-sections, CheckMate demands full simulated events to check for monojet bounds.

We found that all scanned points passed the monojet constraints from CheckMate, but not from searches for hadronic decays of squarks and gluinos. Smodels decomposes the full model into simplified model spectrum topologies taking into account efficiency selection criteria in order to make the correct comparison with its internal database. After that, it seeks for an experimental bound on the cross-section times branching ratio, $\sigma(p p \rightarrow$ $\left.D_{1} D_{2}\right) \times B R\left(D_{1(2)} \rightarrow q+H^{0}\right)$ in our case, from a list of experimental publications and conference notes. Upper limits from those experimental studies on the cross sections, $\sigma_{95 \%}$, at $95 \%$ confidence level (CL), are then compared to the simulated $\sigma\left(p p \rightarrow D_{1} D_{2}\right) \times$ $B R\left(D_{1(2)} \rightarrow q+H^{0}\right)$. A model is considered excluded with CL above $95 \%$, for one or more analysis, whenever we have $\sigma\left(p p \rightarrow D_{1} D_{2}\right) \times B R\left(D_{1(2)} \rightarrow q+H^{0}\right)>\sigma_{95 \%}$, or, in terms of the ratio $r \equiv \frac{\sigma\left(p p \rightarrow D_{1} D_{2}\right) \times B R\left(D_{1(2)} \rightarrow q+H^{0}\right)}{\sigma_{95 \%}}$, if the output is $r \geqslant 1$.

In figure 3, we show some possible scenarios corresponding to particular selections of the parameters of the model relevant for the DM analysis of the next section, where $D$ couples exclusively with either the first family (black lines), the second family (blue lines) or the third family (red lines). For each scenario, the yellow shaded regions above $r=1$ can be 

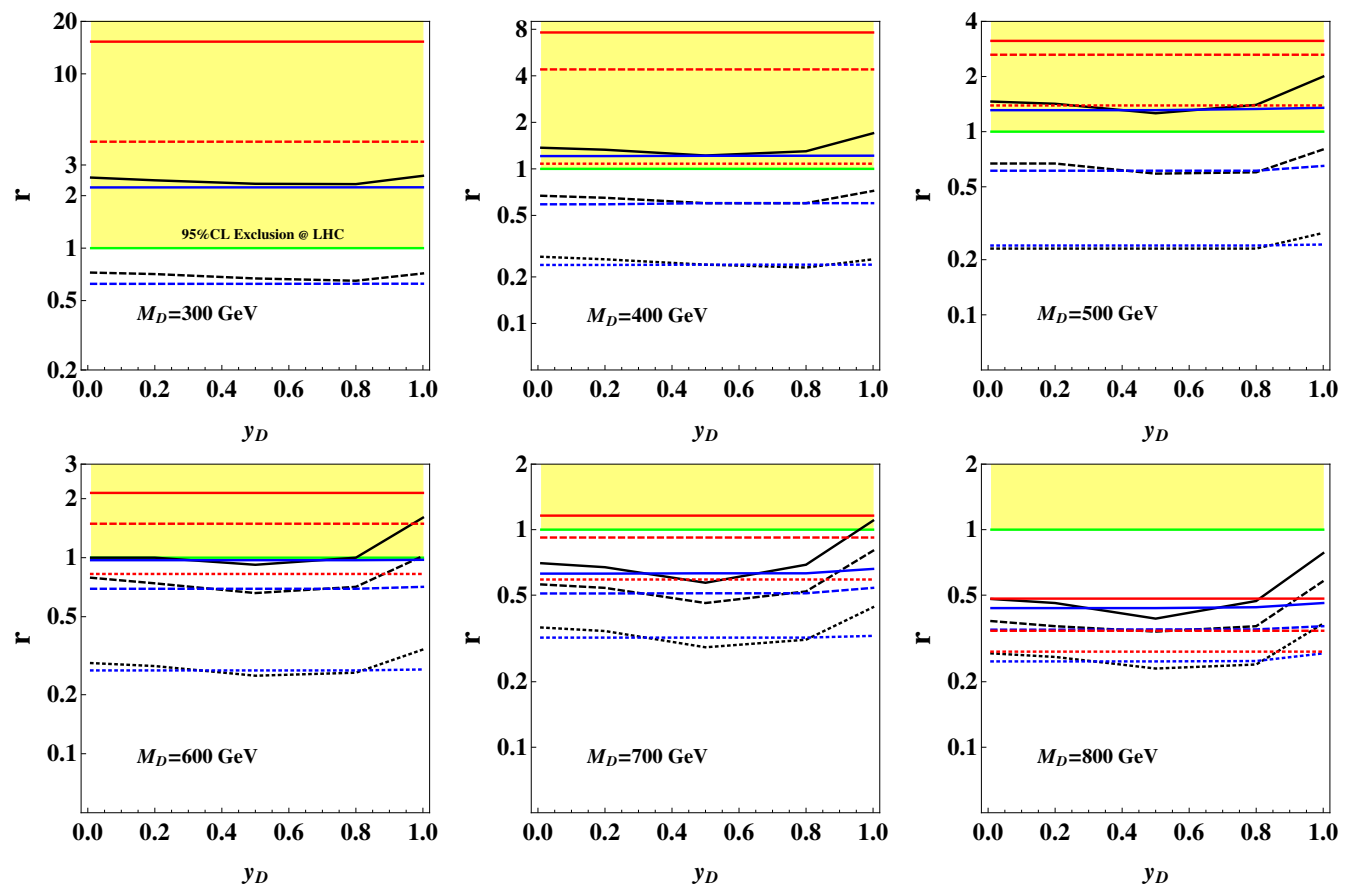

Figure 3. Values of the ratio $r$, defined in the text, as a function of $y_{D}$ for several values of $M_{D}$. The shaded yellow area corresponding to $r \geq 1$ is excluded with $95 \%$ at least. For each $M_{D}$, the solid, dashed and dotted lines correspond to $M_{H^{0}}=100 \mathrm{GeV}, 200 \mathrm{GeV}$, and $300 \mathrm{GeV}$, respectively. In all these scenarios, $M_{H^{ \pm}}=M_{A^{0}}=M_{H^{0}}+5 \mathrm{GeV}$, where black, blue and red lines correspond to $D$ coupling with the first, second and third family, respectively.

considered excluded with 95\% CL, at least. For each $M_{D}$, the solid, dashed and dotted black lines correspond to the scenarios $\left(M_{H^{0}}, M_{A^{0}, H^{ \pm}}\right)=(100,105) \mathrm{GeV},\left(M_{H^{0}}, M_{A^{0}, H^{ \pm}}\right)=$ $(200,205) \mathrm{GeV}$ and $\left(M_{H^{0}}, M_{A^{0}, H^{ \pm}}\right)=(300,305) \mathrm{GeV}$, respectively.

The first observation that we can draw from figure 3 is that the most restrictive scenario occurs when $D$ interacts with the third family. In this case, $D$ has a typical SUSY signature matching with searches for direct production of bottom squark pairs which translates to harder constraints in our case. Second, the bounds for the second and third families are very weakly dependent on the Yukawa coupling, an effect that we have anticipated previously. On the hand, for Yukawa couplings between 0.2 and 0.8, approximately, first family scenarios have smaller $r$ ratios by virtue of the destructive interference between QCD and Yukawa contributions. Although the effect is not so pronounced, for $y_{D} \gtrsim 0.8$ we see a clear trend towards the exclusion region as, in this regime, the production cross section increases.

We also see that, in general, as the mass of $D$ increases the production cross section drops fast as shown in figure 2, but the cut efficiency somewhat compensates for the signal decrease up to $700 \mathrm{GeV}$ approximately as the jets becomes harder. For masses larger than $\sim 700 \mathrm{GeV}$, the production cross section is too low and the model evades the collider constraints unless the Yukawa coupling is larger than 1.

In the next section we present the results of our analysis of the DM candidate of the model taking into account all the collider constraints we obtained. 


\section{Dark matter phenomenology}

Our work is based on a two component DM, videlicet, comprised of a WIMP $\left(H^{0}\right)$ and an axion $(a)$ (see [55-66] for other realizations of WIMP plus axion DM scenario). It is suitable to address the important aspect of the relic density of each component individually before discussing the WIMP+axion scenario. We start by reviewing how the WIMP abundance is obtained.

\subsection{WIMP relic density}

The abundance of the WIMP is obtained in the usual way, by solving the Boltzmann equation, with the help of micrOMEGAS $[67,68]$. In this realization, the WIMP is in thermal equilibrium with SM particles, i.e., the annihilation and production interactions occur at similar rates in the early Universe. Although, as the Universe expands and the temperature drops below the DM mass, they can no longer be produced, and are simply able to pairannihilate. Eventually, the expansion rate equals the rate for pair annihilation and then freeze-out is established. Thus, the larger the annihilation cross section the fewer DM particles were left-over after the freeze-out. From then on, the abundance of left-over DM particle is kept basically constant. This is the standard picture, where no coannihilations are present. For the IDM, this is not the case and coannihilations play a dominant role in the WIMP abundance.

In the IDM the $H^{0}$ pair annihilation into SM particles is of the order of $6 \times 10^{-26} \mathrm{~cm}^{3} / \mathrm{s}$, for $500 \mathrm{GeV}<M_{H}^{0}<3 \mathrm{TeV}$ [69], which would naively produce an abundance below the correct value. Nevertheless, the other inert scalars $H^{ \pm}, A^{0}$ interact at similar rates with $H^{0}$ and SM particles, which makes them freeze-out at a similar time. Since they are not stable, after the freeze-out they decay into $H^{0}$ increasing its abundance to match the correct value. This mechanism was explained in detail recently in [69, 70]. Thus, coannihilations are an important ingredient in the IDM in order to have a viable WIMP. The setup remains identical in the WIMP+axion framework that we will advocate, as long as the coannihilations involving the exotic quark $D$ are suppressed (to be considered in section 4.4) and axions have an insignificant relic density.

The IDM DM phenomenology can be wisely split into three mass regimes [12, 28, 35, 71]

Low mass: $\boldsymbol{M}_{\boldsymbol{H}^{\mathrm{o}}}<\boldsymbol{M}_{\boldsymbol{W}}$. In this mass range the model resembles the singlet scalar Higgs portal DM where the main annihilation modes are into light fermions, mainly $b b$ quarks, with annihilations controlled by the quartic coupling that mix the SM Higgs and $H^{0}$ [72-93]. There, $A^{0}$ and $H^{ \pm}$have to decouple in order to avoid direct and indirect DM WIMP searches. In summary, one needs to live at the Higgs resonance to be able to reproduce the right relic density while avoiding existing constraints [94].

Heavy dark matter: $\boldsymbol{M}_{\boldsymbol{H}^{\mathbf{0}}}>\mathbf{5 0 0} \mathbf{G e V}$. This mass region is viable and consistent with direct, indirect and collider searches. It can reproduce the right relic density thanks to coannihilations effects involving the inert scalars as we mentioned earlier, with a mass splitting $100 \mathrm{KeV}<M_{H^{0}}-M_{H^{+}, A^{0}}<15 \mathrm{GeV}$ [35]. For recent studies in this mass region we 
refer to [69, 70]. Interestingly, almost the entire parameter space of the model is expected to be probed by the Cherenkov Telescope Array [69, 70].

Intermediate mass: $M_{W}<M_{H^{0}}<500 \mathrm{GeV}$. This mass region has been entirely excluded in the light of recent direct detection limits and relic density constraints [14]. Here, the annihilation rate into gauge bosons is very efficient leading to a dwindled relic density. It is in this precise mass region which the two component DM scenario we are advocating is most relevant. Since the WIMP share its duties with the axion, the constraints are relaxed and the total relic density of $\Omega_{\text {total }}=0.1$ can be achieved, motivating our work. We will explicitly show further how this is realized.

\subsection{Axion relic density}

As for the axion, the key question turns out to be, when is the Peccei-Quinn symmetry broken: before or after the inflation period? If it is broken before the end of inflation, the only process relevant for axion production is coherent oscillation due to the vacuum realignment and the axion relic density is given by [11,31],

$$
\Omega_{a} h^{2} \sim 0.18 \theta^{2}\left(\frac{f_{a}}{10^{12} \mathrm{GeV}}\right)^{1.19}
$$

where $\theta$ is the initial axion misalignment angle. Note that, if $\theta$ is of order of unity, the axion can reproduce the total relic density, $\Omega_{a} h^{2} \sim \Omega h^{2}$, only for $f_{a} \sim 10^{12} \mathrm{GeV}$. We will set $\theta=1$ throughout.

In summary, the total relic density is given by $\Omega h^{2}=\Omega_{H^{0}} h^{2}+\Omega_{a} h^{2}$, where $\Omega_{H^{0}} h^{2}$ is the relic density due to the WIMP, and $\Omega_{a} h^{2}$ the one corresponding to the axion, which depends on the cosmological model. That said, it is a good timing to discuss the two component DM abundance in more quantitative terms.

\subsection{Mixed WIMP-axion dark matter in the IDM}

In order to take into account both axion and WIMP contributions to the total observed relic density $^{5}$ we have scanned the free parameter space in the range shown in table 2, always enforcing $M_{A^{0}}-M_{H^{0}} \gtrsim 100 \mathrm{keV}$ to avoid the ruled out ineslatic DM regime [96, 97]. The result of this scan is displayed in figure 4. There we show the relative WIMP and axion contributions to the total abundance as a function of $f_{a}$. In figure 4 we have assumed that the exotic $D$ quark couples only to one family of SM quarks at a time through $y_{D}$, and concluded that the results are basically identical with a mild difference, within $3 \%$, for the third family, as one can see in figure 2.

There important remarks are in order:

(i) We can clearly see that for $f_{a} \lesssim 5 \times 10^{10} \mathrm{GeV}$, we enter the WIMP dominated regime.

(ii) For $5 \times 10^{10} \mathrm{GeV} \lesssim f_{a} \lesssim 7 \times 10^{11} \mathrm{GeV}$, we have a plausible two component DM setup being able to meet $\Omega_{\text {total }} h^{2}=0.12$.

(iii) For $f_{a}>7 \times 10^{11} \mathrm{GeV}$, we go into the axion dominated scenario.

\footnotetext{
${ }^{5}$ To calculate the WIMP contribution, we have implemented the model in FeynRules [52] and used microMEGAs [95].
} 


\begin{tabular}{|c|c|}
\hline Parameter & Scan range \\
\hline$M_{H^{0}}$ & $60-10^{3} \mathrm{GeV}$ \\
$M_{A^{0}}-M_{H^{0}}$ & $0-10 \mathrm{GeV}$ \\
$M_{H^{ \pm}}-M_{H^{0}}$ & $0-10 \mathrm{GeV}$ \\
$\lambda_{L}$ & $10^{-3}-1$ \\
$M_{D}-M_{H^{0}}$ & $0-10^{3} \mathrm{GeV}$ \\
$y_{D}$ & $10^{-2}-1$ \\
$f_{a}$ & $10^{9}-10^{15} \mathrm{GeV}$ \\
\hline
\end{tabular}

Table 2. Parameter range used for the DM scan.

This plot visibly proves that one can successfully have a two component DM in the model. However, an important information in this two component DM scenario is the WIMP mass. That said, we display in figure 5 the fractions $R_{X}$, with $X=H^{0}$, a, of the total relic density as a function of the Peccei-Quinn scale $f_{a}$ explicitly showing the DM mass encoded in the curves. The fraction of relic abundance is defined as

$$
R_{X}=\frac{\Omega_{X} h^{2}}{\Omega h^{2}} .
$$

We have imposed $M_{D}>300 \mathrm{GeV}$ and the misalignment angle $\theta=1$. In addition, we have also considered the constraints (2.16) discussed in the end of section 2 and the restrictions showed in figure 3 . The curve starting at $R_{X}>80 \%$ represents the inert scalar $H^{0}$ abundance, while the curve starting at $R_{X}<20 \%$ reflects the axion's. We enforced the total relic density to be $\Omega h^{2} \sim 0.1199 \pm 0.0027$ [98] throughout. We see clearly in figure 5 that the WIMP dominated regime favors heavier masses $\left(M_{H^{0}}>400 \mathrm{GeV}\right)$, whereas the axion dominated one prefers $M_{H^{0}}<280 \mathrm{GeV}$. The reason why the WIMP dominated region prefers heavier masses is just a consequence of the IDM nature of the WIMP, since it is well known that for $M_{W}<M_{H^{0}}<500 \mathrm{GeV}$ the WIMP cannot produce $\Omega h^{2} \sim 0.1199 \pm 0.0027$. As aforementioned, this is no longer problematic in the light of our two component DM where the axion abundance makes up for the deficit, depending on the value of $f_{a}$.

In figure 4 the WIMP can account for $100 \%$ of the relic density as $f_{a}$ drops well below $10^{10} \mathrm{GeV}$, because there we entered in the mass region $M_{H^{0}}>500 \mathrm{GeV}$ where the relic density constraint is satisfied. The heavy quarks also play a role in setting the WIMP abundance through coannihilation processes, when $M_{D} \sim M_{H^{0}}$, as we will investigate in detail further.

\subsection{New coannihilations with vector-like quarks}

The DM phenomenology of the IDM from Peccei-Quinn symmetry differs from the IDM in two fundamental ways: (i) the presence of coannihilations involving the heavy vector-like quarks (D); (ii) the axion now contributes to the total relic density.

The new coannihilation processes involving the initial states $H^{0} D, A^{0} D, H^{ \pm} D$ and $\bar{D} D$, will appear mediated by the Yukawa coupling $y_{D}$. Such coannihilations are exponentially suppressed by the mass splitting $\Delta M \equiv M_{D}-M_{H^{0}}$, and proportional to the Yukawa 


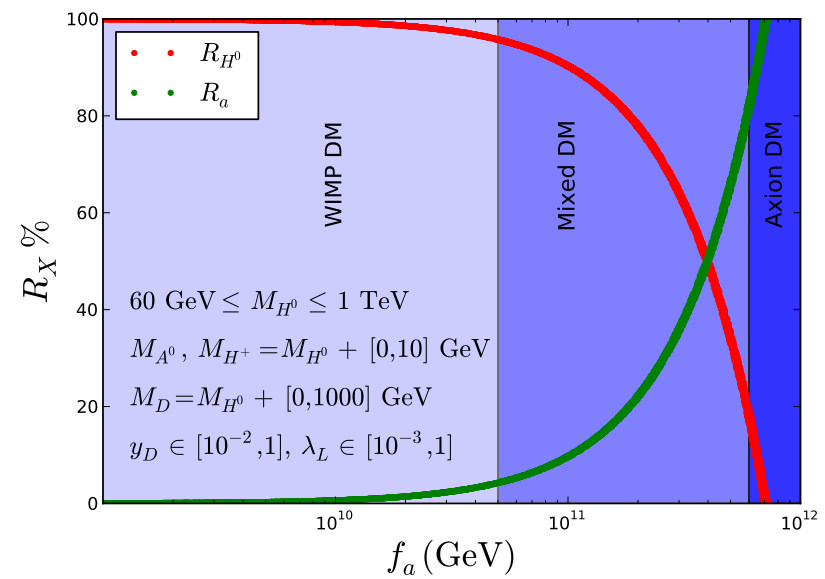

Figure 4. Contributions to the total relic density $\Omega h^{2} \sim 0.1199 \pm 0.0027$ [98] as a function of the PQ scale $f_{a}$. The plot is the similar for our scenario in relation to the one presented in ref. [15]. We have assumed $M_{D}>300 \mathrm{GeV}, \theta=1$, and the restrictions in eq. (2.16). The reason $H^{0}$ can meet the correct abundance is due to coannihilations involving the heavy vector-like quark.

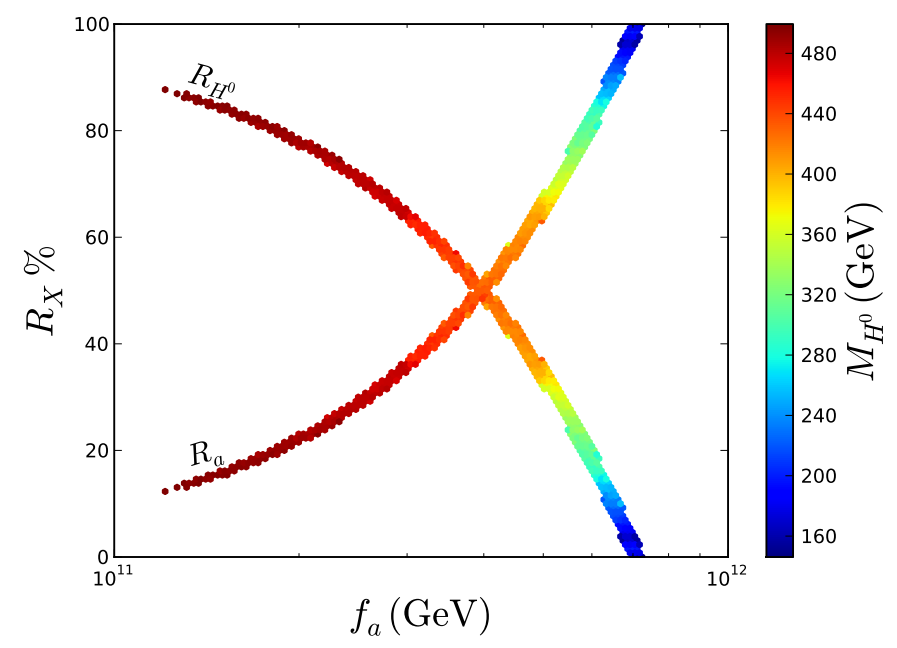

Figure 5. Relative contribution of the inert scalar $H^{0}$ and axion to the total relic density, defined as $R_{X}$, as a function of $f_{a}$. The curve starting at $R_{X}>80 \%$ represents the inert scalar $H^{0}$ abundance, while the curve starting at $R_{X}<20 \%$ reflects the axion. We enforced the total relic density to be $\Omega h^{2} \sim 0.1199 \pm 0.0027$ [98] throughout. We have assumed $M_{D}>300 \mathrm{GeV}, \theta=1$, and the restrictions in eq. (2.16). 
coupling $y_{D}$. If the mass difference is sufficiently large or the Yukawa coupling is dwindled, the $H_{0}$ phenomenology remains identical to the IDM. To quantify the impact of these new coannihilation processes on the WIMP relic density of the IDM from Peccei-Quinn symmetry, we have used the scan over the free parameters showed in table 2. We have found that the coannihilation processes with the exotic quark $D$ are negligible when $M_{D} \gtrsim 1.2 M_{H^{0}}$ and $y_{D} \lesssim 0.7$, so that we recover the DM phenomenology of the IDM in such a case, even though the coannihilation process $\bar{D} D \rightarrow g g$ has pure gauge contributions independently of the Yukawa $y_{D}$.

Generally speaking, coannihilation processes such as these only play a role if the mass splitting between the WIMP and the other odd particles is within 10-15\%, due to the Boltzmann suppression, which is the reason for negligible coannihilation processes when $M_{D} \gtrsim 1.2 M_{H^{0}}$.

We display in figure 6 the WIMP relic density as a function of $M_{H^{0}}$ for the mass differences $\Delta M=10 \mathrm{GeV}$ (blue line), $50 \mathrm{GeV}$ (yellow line), $100 \mathrm{GeV}$ (green line), $200 \mathrm{GeV}$ (red line) and for two values of the Yukawa coupling $y_{D}=0.5$ (left panel) and $y_{D}=1$ (right panel). The dashed line correspond to the decoupled limit, $M_{D} \gg M_{H^{0}}$, where the coannihilations are negligible and the IDM phenomenology is recovered. The horizontal blue band correspond to the current bound $\Omega h^{2} \sim 0.1199 \pm 0.0027$ [98].

Note that the coannihilations with the exotic quark decrease the WIMP population and increase the allowed DM mass compatible with the data. That is because the inclination of the relic density curve of $H^{0}$ depends on how efficient vector-quark coannihilations are. Thus, once we reach the overabundant regime, we can simply turn on such coannihilation by increasing $y_{D}$ and making the mass difference smaller, and bring down the abundance to the correct vale. In other words, we simply change the inclination of the abundance curves as can be explicitly seen in figure 6 .

In particular, for $y_{D}=1$, right panel of figure 6 , we can see a significant difference between the case in which $\Delta M=200 \mathrm{GeV}$ (red line), where the WIMP reproduce the total relic density for $M_{H^{0}} \approx 800 \mathrm{GeV}$, and the case in which $\Delta M=100 \mathrm{GeV}$ (green line), where the WIMP reproduce the total relic density for a larger mass of $M_{H^{0}} \approx 900 \mathrm{GeV}$. It is only for a splitting $\Delta M>200 \mathrm{GeV}$ that our model recovers the IDM phenomenology, where the vector-like quark coannihilations are turned off. For $y_{D}=0.5$ this mass difference is $\Delta M>100 \mathrm{GeV}$. Notice that for $y_{D}=1$, the coannihilation cross sections are larger and hence a mass splitting must be mildly larger compared to the case with $y_{D}=0.5$ in order to suppress the coannihilations, where $\Delta M>100 \mathrm{GeV}$ suffices.

In the collider section we observed that $y_{D}>0.8$ might be problematic due to monojet and dijet plus missing energy constraints, therefore $y_{D}=0.5$ is a feasible benchmark model, where both relic density and collider constraints are satisfied as well as the direct and indirect DM detection probes addressed in the following.

\subsection{Direct detection}

WIMPs might also scatter off of nuclei and deposit an energy which can be measured by underground detectors such as LUX [99], CDMS [100] and PICO [101] among others [102107], all of them using different target nuclei and readout techniques. By discriminating 

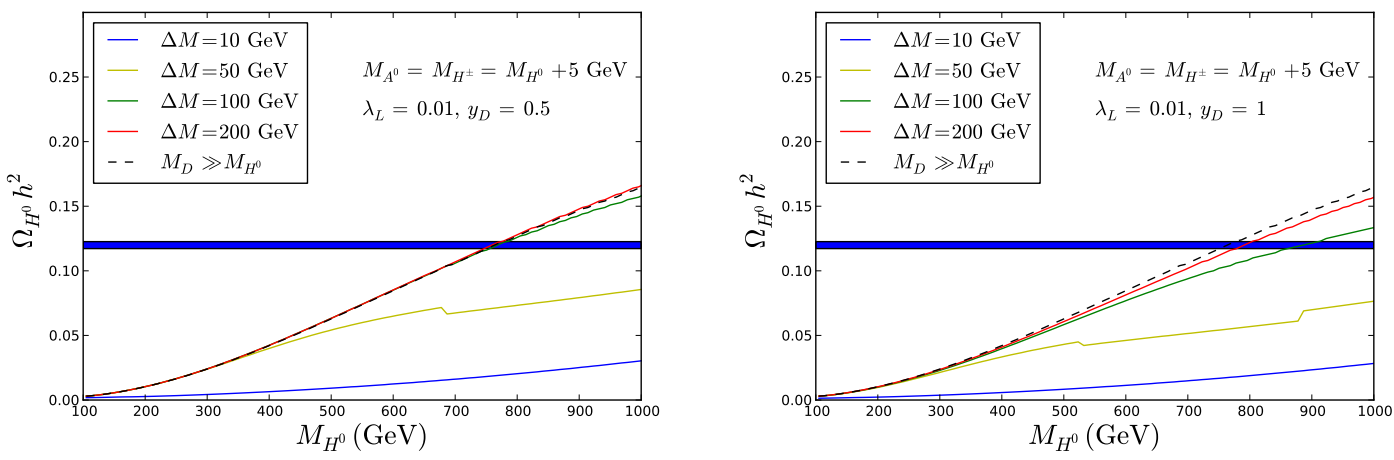

Figure 6. WIMP relic density as a function of $M_{H^{0}}$ for different values of $\Delta M \equiv M_{D}-M_{H^{0}}$ and for $y_{D}=0.5$ (left panel) and $y_{D}=1$ (right panel). The horizontal blue line correspond to the actual experimental bound $\Omega h^{2} \sim 0.1199 \pm 0.0027$ [98]. The decoupling limit, $M_{D} \gg M_{H^{0}}$, coincides with the inert doublet model.

nuclear recoil from electron recoils, the experiments have been able to place stringent limits in the scattering cross section vs WIMP mass, capable of depositing an energy above few $\mathrm{keV}$. In the IDM model, the direct detection limits from LUX, which is currently the world leading experiment, can be easily evaded by requiring the mass splitting between $A^{0}$ and $H^{0}$ to be above $100 \mathrm{KeV}$, and the coupling $\lambda_{L}$ to be suppressed with no prejudice to our reasoning. In particular, the values $\left|\lambda_{L}\right| \lesssim 0.01$ are well below the current sensitivity of LUX [94] and also the projected sensitivity of XENON1T [108, 109].

In our model augmenting the IDM, we need to consider the presence of the exotic quark $D$ which can mediate the WIMP interaction with the nucleus by s-channel and tchannel scattering with quarks/gluons, as shown in figure 1, diagrams (g) and (h). ${ }^{6}$ Such interactions are governed by the Yukawa coupling $y_{D}$ and the exotic quark mass $M_{D}$. When $M_{D} \sim M_{H^{0}}$, which is of interest to us since coannihilations with the $D$-quark become important, there is an enhancement in the cross section as a result of the inelastic regime. Taking $y_{D} \lesssim 0.5$, the model is consistent with the LUX bound on the spinindependent scattering cross section. Thus, from the left panel of figure 6 , we can see that the model can simultaneously yield the right abundance and accommodate the LUX limit. For $M_{D} \sim 1.2 M_{H^{0}}\left(\Delta M \sim 0.2 M_{H^{0}}\right)$, when coannihilations are turned off, we find that for $y_{D} \lesssim 0.7$, the model is below LUX and future XENON1T [108] bounds. In summary, our benchmark model with $y_{D}=0.5$ is perfectly consistent with current and projected limits from direct detection.

Thus we conclude that the right panel in figure 6 , where $y_{D}=1$, is excluded in the light of direct detection experiments. This conclusion shows the high degree of DM complementarity in our model. However, this holds true as long as $H^{0}$ accounts for the total DM abundance, which is not necessarily true in our model, specially when $M_{W}<$ $M_{H^{0}}<500 \mathrm{GeV}$. Since the direct detection limits are linearly proportional to the WIMP

\footnotetext{
${ }^{6} \mathrm{~A}$ study at one loop was realized in [110] for the singlet scalar model augmented with a exotic quark and neglecting the Higgs portal.
} 
local density, the bounds are alleviated and the model can be made compatible with direct detection in the regime where the axion makes up a large fraction of the abundance, i.e., for $f_{a} \gtrsim 7 \times 10^{11} \mathrm{GeV}$. We handpicked these two values for $y_{D}$ to show precisely when direct detection constraints become relevant and how our two component DM scenario plays an important role in satisfying both relic density and direct detection searches for WIMPs.

\subsection{Indirect detection}

WIMPs may self-annihilate producing a sizable amount of gamma-rays and cosmic-rays over the astrophysical background (see [111-113] for recent reviews). Searches for WIMP annihilations have been performed in several target regions such as the Galactic Center, Dwarf Galaxies, Cluster of Galaxies etc [69, 114-125]. In our model the mass of interest is hardly touched by current Fermi-LAT and H.E.S.S. limits [126], since the need for the axion to complement the WIMP under-abundance relaxes the indirect detection limits which depend on the local DM density squared.

Even assuming that $H^{0}$ makes up the entire DM of the Universe, for $500 \mathrm{GeV}<$ $M_{H^{0}}<3 \mathrm{TeV}$, Fermi-LAT limits are rather weak, with H.E.S.S. ruling just a tiny fraction of the parameter space [69], unless boost factors are advocated [70]. It is worth mentioned that the Cherenkov Telescope Array might improve existing limits in more than one order of magnitude, and depending on the level of systematics uncertainties achieved [127, 128], the entire model below $3 \mathrm{TeV}$ might be excluded [69]. We emphasize though, that in our two component DM scenario such conclusions are strongly relaxed. In other words, our results are consistent with exclusion limits from indirect DM detection searches.

\section{Conclusions}

Since WIMPs and axions are arguably the most compelling DM candidates in the literature, we investigate the possibility of two component DM in a well motivated model, namely the Inert Doublet Model. We present a model that contains, beyond the SM fields, a scalar inert doublet, a scalar singlet hosting an axion, and a new vector-like quark $D$. These fields allow an implementation of the Peccei-Quinn U(1) $P Q$ symmetry that solves the strong CP problem and gives rise to an invisible axion. The inert doublet originates a candidate for dark matter, stabilized by a natural $\mathbb{Z}_{2}^{D}$ symmetry remnant from the breakdown of $\mathrm{U}(1)_{P Q}$ symmetry following ref. [15]. The new quark may provide a new portal to connect the SM to the dark sector, which is comprised of particles that are odd under $\mathbb{Z}_{2}^{D}$ transformations plus the axion.

Along with the WIMP, the new quark gives rise to signals involving jets plus missing energy, and also monojets at the LHC. In order to investigate possible restrictions on the parameter space of the model, we have studied all these potential signals at the LHC considering that the $D$ quark couples to the WIMP and with just one of the SM families. We found that the most restrictive scenario occurs when $D$ quark couples to the third family bottom quark. For example, such a scenario is excluded at $95 \%$ C.L for masses of the scalars $H^{0}, A^{0}$, and $H^{ \pm}$being $\left(M_{H^{0}}, M_{A^{0}, H^{ \pm}}\right) \leq(200,205) \mathrm{GeV}$, if $M_{D} \leq 600 \mathrm{GeV}$ and $y_{D} \leq 1$. In the case where the $D$ quark couples with the first or the second family, 
the restrictions are milder, and masses $\left(M_{H^{0}}, M_{A^{0}, H^{ \pm}}\right) \geq(200,205) \mathrm{GeV}$ are allowed for $M_{D} \geq 400 \mathrm{GeV}$ for all Yukawa couplings up to at least unity.

In our model, DM is composed by two components, the lightest inert scalar $\left(H^{0}\right)$ and the axion. Within this scenario we performed an investigation on how the fractions of the DM relic abundance corresponding to the WIMP and to the axion change depending on the scale $f_{a}$ of the breakdown of the $\mathrm{U}(1)_{P Q}$ symmetry, the mass of the WIMP, the masses of the other particles odd by the $\mathbb{Z}_{2}^{D}$ symmetry. For example, for values $f_{a} \leq 10^{10} \mathrm{GeV}$ the WIMP would constitute essentially all the DM, with the axion being an irrelevant fraction of it. As $f_{a}$ increases the axion relic density raises, reaching a value equal to the WIMP relic density for $f_{a} \simeq 4 \times 10^{11} \mathrm{GeV}$.

In contrast with the inert Higgs doublet model, we found that in our model it is possible to have the WIMP from the inert doublet with mass in the interval $100 \mathrm{GeV} \lesssim$ $M_{H^{0}} \lesssim 500 \mathrm{GeV}$, and comprising only a fraction of the total DM relic abundance. This region is phenomenologically important for direct detection experiments and LHC searches of exotic quarks and DM. In particular, we have shown that the IDM phenomenology remains unchanged when the coannihilations effects with the exotic quark are negligible and this happens for $M_{D} \gtrsim 1.2 M_{H^{0}}$. We conclude that one can have a plausible two component DM (WIMP plus axion) satisfying the relic density as well as collider, direct and indirect DM detection constraints.

\section{Prospects}

The assumption that the DM is composed by two or more type of particles impacts on the experiments searching for WIMPS and axions. For example, if the axion relic density constitute an irrelevant fraction of the DM the axion could not be direct detected in haloscopes experiments [129], but it could still be accessible in the projected experiment IAXO [130], which arises as a promising laboratory to test the model we proposed. On the WIMP side, direct future experiments with large exposure such as XENON1T [109] and LZ [131] are quite desired. Future collider constraints stemming from a possible $100 \mathrm{TeV}$ collider or linear collider might also constrain the model even further [132-136].

\section{Acknowledgments}

A. Alves, A.G. Dias and C.C. Nishi acknowledge financial support from the Brazilian CNPq, processes 307098/2014-1, 303094/2013-3 and 311792/2012-0, respectively, and FAPESP, process 2013/22079-8 (A.A., A.G.D., C.C.N.). D. Camargo thanks CAPES for financial support. R. Longas is supported by COLCIENCIAS and acknowledges the hospitality of Universidade Federal do ABC in the early stage of this work. F. S. Queiroz is grateful to the Mainz Institute for Theoretical Physics (MITP) for its hospitality and its partial support during the completion of this work. 


\begin{tabular}{|c|c|c|c|c|c|}
\hline & $D_{L}$ & $D_{R}$ & $H_{D}$ & $S$ & $\varphi$ \\
\hline $\mathrm{U}(1)_{P Q}$ & -1 & 1 & -1 & 2 & 1 \\
\hline
\end{tabular}

Table 3. Fields with nonzero PQ charges.

\section{A Simple UV completions}

\section{A.1 $U(1)_{P Q}$ breaking in the Higgs potential}

It is natural to expect that the $\mathrm{U}(1)_{\mathrm{PQ}}$ breaking at the high scale (larger than $10^{9} \mathrm{GeV}$ ) induces at lower energies the operator in (2.5). We present here a simple model where that happens.

To complete the model, we add another SM singlet scalar $\varphi$ with PQ charge unity but inert (no vev). The relevant terms in the Lagrangian above the PQ scale will be

$$
\begin{aligned}
\mathcal{L} \supset & \bar{q}_{L} H_{D} D_{R}+S^{*} \bar{D}_{L} D_{R}+\varphi^{*} \bar{D}_{L} d_{R} \\
& +S^{*} \varphi^{2}+\left(H^{\dagger} H_{D}\right) \varphi+\left(H^{\dagger} H_{D}\right) \varphi^{*} S+\text { h.c. }
\end{aligned}
$$

We omit the coefficients for simplicity and, for definiteness, we take the exotic quark to be of charge $-1 / 3$, denoting it by $D$. The case of charge $2 / 3$ is analogous. The PQ charges are given in table 3 .

After $S$ acquires a vev $\langle S\rangle$, the breaking $\mathrm{U}(1)_{\mathrm{PQ}} \rightarrow \mathbb{Z}_{2}^{D}$ is induced and we get effectively

$$
\begin{aligned}
\mathcal{L} \supset & y_{D}^{i} \bar{q}_{i L} H_{D} D_{R}+M_{D} \bar{D}_{L} D_{R}+\kappa_{j}^{*} \varphi^{*} \bar{D}_{L} d_{j R} \\
& +\mu_{\varphi}^{2} \varphi^{2}+\mu_{H}\left(H^{\dagger} H_{D}\right) \varphi+\mu_{H}^{\prime}\left(H^{\dagger} H_{D}\right) \varphi^{*}+\lambda_{\varphi} \varphi^{4}+\text { h.c. }
\end{aligned}
$$

We assume $\sqrt{\left|\mu_{\varphi}^{2}\right|}$ and the mass accompanying $|\varphi|^{2}$ to be much smaller than the PQ scale but much larger than the electroweak scale. The $\varphi^{2}$ term splits the complex scalar into two real scalars $\varphi_{1}, \varphi_{2}$ of different masses $M_{1}, M_{2}$. Thus the terms with $\mu_{H}, \mu_{H}^{\prime}$ of (A.2), which can be recast in the form

$$
\left(H^{\dagger} H_{D}\right)\left(\mu_{1} \varphi_{1}+i \mu_{2} \varphi_{2}\right)
$$

leads to the desired operator (2.5) with coefficient

$$
\lambda_{5}=\left(\frac{\mu_{1}^{2}}{M_{1}^{2}}-\frac{\mu_{2}^{2}}{M_{2}^{2}}\right) .
$$

This model at the PQ scale is identical to the model I presented in ref. [15] which realizes $\mathrm{U}(1)_{\mathrm{PQ}} \rightarrow \mathbb{Z}_{2}^{D}$ in a KSVZ type axion model and, additionally, also generates neutrino masses radiatively. At the $\mathrm{TeV}$ scale, however, our focus is on a different physical spectrum where the DM candidates are the axion and the lightest neutral member of the inert doublet while the interaction of the heavy quark with the SM occurs also through the inert doublet. We should also emphasize that a different realization may lead to the same physical spectrum at the $\mathrm{TeV}$ scale - the SM augmented by an inert doublet, an axion and a exotic quark — but to a different particle content at the PQ scale. 


\section{A.2 Lighter exotic quark mass}

For the model (A.2), it is expected that the exotic quark mass $M_{D}$ be at the order of the PQ breaking scale or at most few orders of magnitudes lower. To get $M_{D}$ at the $\mathrm{TeV}$ scale one has to tune the Yukawa coupling to at least 6 orders of magnitude. Here we show a variant where the exotic $D$ quark have mass decoupled from the PQ scale and thus can be lighter.

The variant includes another exotic quark, which we keep denoting as $D$, while the original exotic quark is renamed as $D^{\prime}$. Thus the new exotic quark $D$ has the same quantum numbers as $D^{\prime}$ except that it is vector-like with respect to PQ symmetry: $\mathrm{PQ}\left(D_{L}\right)=$ $\operatorname{PQ}\left(D_{R}\right)=1$. Now $D$ is still the quark that couples to the SM quarks but the QCD anomaly is generated by $D^{\prime}$.

The relevant Lagrangian is modified to

$$
\mathcal{L} \supset \bar{q}_{L} H_{D} D_{R}+S^{*}{\overline{D^{\prime}}}_{L} D_{R}^{\prime}+S^{*}{\overline{D^{\prime}}}_{L} D_{R}+\bar{D}_{L} D_{R}^{\prime}+\bar{D}_{L} D_{R}+\text { h.c. }
$$

After PQ breaking we get

$$
\mathcal{L} \supset M_{D^{\prime} D^{\prime}}{\overline{D^{\prime}}}_{L} D_{R}^{\prime}+M_{D^{\prime} D}{\overline{D^{\prime}}}_{L} D_{R}+\tilde{M}_{D D^{\prime}} \bar{D}_{L} D_{R}^{\prime}+\tilde{M}_{D D} \bar{D}_{L} D_{R}+\text { h.c. },
$$

where the coefficients are now explicitly written and the masses denoted by tilde are bare and in principle can be much smaller than the PQ scale.

We can write

$$
\mathbb{M}_{D}=\left(\begin{array}{ll}
\tilde{M}_{D D} & \tilde{M}_{D D^{\prime}} \\
M_{D^{\prime} D} & M_{D^{\prime} D^{\prime}}
\end{array}\right) .
$$

It is easy to see for the case of $\tilde{M}_{A B} \ll M_{A B}, A, B=D, D^{\prime}$, that $U_{L}$ diagonalizing $\mathbb{M}_{D} \mathbb{M}_{D}^{\dagger}$ has a small mixing angle while $U_{R}$ diagonalizing $\mathbb{M}_{D} \mathbb{M}_{D}^{\dagger}$ has a large mixing angle. After, integrating out the heaviest state, we end up with a lighter exotic quark with mass $M_{D} \sim O\left(\tilde{M}_{A B}\right)$ with appreciable coupling to the SM quarks through the first term of (A.5).

Open Access. This article is distributed under the terms of the Creative Commons Attribution License (CC-BY 4.0), which permits any use, distribution and reproduction in any medium, provided the original author(s) and source are credited.

\section{References}

[1] S. Weinberg, A New Light Boson?, Phys. Rev. Lett. 40 (1978) 223 [inSPIRE].

[2] F. Wilczek, Problem of Strong $p$ and $t$ Invariance in the Presence of Instantons, Phys. Rev. Lett. 40 (1978) 279 [INSPIRE].

[3] R.D. Peccei and H.R. Quinn, CP Conservation in the Presence of Instantons, Phys. Rev. Lett. 38 (1977) 1440 [INSPIRE].

[4] J.E. Kim and G. Carosi, Axions and the Strong CP Problem, Rev. Mod. Phys. 82 (2010) 557 [arXiv: 0807.3125] [INSPIRE].

[5] J. Jaeckel and A. Ringwald, The Low-Energy Frontier of Particle Physics, Ann. Rev. Nucl. Part. Sci. 60 (2010) 405 [arXiv: 1002.0329]. 
[6] A. Ringwald, Exploring the Role of Axions and Other WISPs in the Dark Universe, Phys. Dark Univ. 1 (2012) 116 [arXiv:1210.5081] [INSPIRE].

[7] J.E. Kim, Weak Interaction Singlet and Strong CP Invariance, Phys. Rev. Lett. 43 (1979) 103 [INSPIRE].

[8] M.A. Shifman, A.I. Vainshtein and V.I. Zakharov, Can Confinement Ensure Natural CP Invariance of Strong Interactions?, Nucl. Phys. B 166 (1980) 493 [INSPIRE].

[9] M. Dine, W. Fischler and M. Srednicki, A Simple Solution to the Strong CP Problem with a Harmless Axion, Phys. Lett. B 104 (1981) 199 [inSPIRE].

[10] A.R. Zhitnitsky, On Possible Suppression of the Axion Hadron Interactions (in Russian), Sov. J. Nucl. Phys. 31 (1980) 260 [inSPIRE].

[11] P. Sikivie, Axion Cosmology, Lect. Notes Phys. 741 (2008) 19 [astro-ph/0610440].

[12] L. Lopez Honorez, E. Nezri, J.F. Oliver and M.H.G. Tytgat, The Inert Doublet Model: An Archetype for Dark Matter, JCAP 02 (2007) 028 [hep-ph/0612275] [INSPIRE].

[13] L. Lopez Honorez and C.E. Yaguna, The inert doublet model of dark matter revisited, JHEP 09 (2010) 046 [arXiv: 1003.3125] [INSPIRE].

[14] L. Lopez Honorez and C.E. Yaguna, A new viable region of the inert doublet model, JCAP 01 (2011) 002 [arXiv: 1011.1411] [INSPIRE].

[15] B. Dasgupta, E. Ma and K. Tsumura, Weakly interacting massive particle dark matter and radiative neutrino mass from Peccei-Quinn symmetry, Phys. Rev. D 89 (2014) 041702 [arXiv: 1308.4138] [INSPIRE].

[16] M. Kadastik, K. Kannike and M. Raidal, Matter parity as the origin of scalar Dark Matter, Phys. Rev. D 81 (2010) 015002 [arXiv:0903.2475] [INSPIRE].

[17] M. Kadastik, K. Kannike and M. Raidal, Dark Matter as the signal of Grand Unification, Phys. Rev. D 80 (2009) 085020 [Erratum ibid. D 81 (2010) 029903] [arXiv:0907.1894] [INSPIRE].

[18] M. Frigerio and T. Hambye, Dark matter stability and unification without supersymmetry, Phys. Rev. D 81 (2010) 075002 [arXiv:0912.1545] [INSPIRE].

[19] N. Nagata, K.A. Olive and J. Zheng, Weakly-Interacting Massive Particles in Non-supersymmetric SO(10) Grand Unified Models, JHEP 10 (2015) 193 [arXiv: 1509.00809] [INSPIRE].

[20] C. Arbelaez, R. Longas, D. Restrepo and O. Zapata, Fermion dark matter from $\mathrm{SO}(10)$ GUTs, Phys. Rev. D 93 (2016) 013012 [arXiv: 1509.06313] [INSPIRE].

[21] S.M. Boucenna, M.B. Krauss and E. Nardi, Dark matter from the vector of SO (10), Phys. Lett. B 755 (2016) 168 [arXiv: 1511.02524] [INSPIRE].

[22] J. Heeck and S. Patra, Minimal Left-Right Symmetric Dark Matter, Phys. Rev. Lett. 115 (2015) 121804 [arXiv: 1507.01584] [INSPIRE].

[23] A.G. Dias, A.C.B. Machado, C.C. Nishi, A. Ringwald and P. Vaudrevange, The Quest for an Intermediate-Scale Accidental Axion and Further ALPs, JHEP 06 (2014) 037 [arXiv: 1403.5760] [INSPIRE].

[24] A. Ringwald and K. Saikawa, Axion dark matter in the post-inflationary Peccei-Quinn symmetry breaking scenario, Phys. Rev. D 93 (2016) 085031 [arXiv:1512.06436] [INSPIRE].

[25] Y. Mambrini, S. Profumo and F.S. Queiroz, Dark Matter and Global Symmetries, Phys. Lett. B 760 (2016) 807 [arXiv: 1508.06635] [INSPIRE]. 
[26] N.G. Deshpande and E. Ma, Pattern of Symmetry Breaking with Two Higgs Doublets, Phys. Rev. D 18 (1978) 2574 [INSPIRE].

[27] E. Ma, Verifiable radiative seesaw mechanism of neutrino mass and dark matter, Phys. Rev. D 73 (2006) 077301 [hep-ph/0601225] [INSPIRE].

[28] R. Barbieri, L.J. Hall and V.S. Rychkov, Improved naturalness with a heavy Higgs: An alternative road to LHC physics, Phys. Rev. D 74 (2006) 015007 [hep-ph/0603188] [INSPIRE].

[29] G. Raffelt and D. Seckel, Bounds on Exotic Particle Interactions from SN 1987a, Phys. Rev. Lett. 60 (1988) 1793 [INSPIRE].

[30] R. Essig et al., Working Group Report: New Light Weakly Coupled Particles, arXiv: 1311.0029 [INSPIRE].

[31] K.J. Bae, J.-H. Huh and J.E. Kim, Update of axion CDM energy, JCAP 09 (2008) 005 [arXiv:0806.0497] [INSPIRE].

[32] L. Visinelli and P. Gondolo, Dark Matter Axions Revisited, Phys. Rev. D 80 (2009) 035024 [arXiv:0903.4377] [INSPIRE].

[33] O. Wantz and E.P.S. Shellard, Axion Cosmology Revisited, Phys. Rev. D 82 (2010) 123508 [arXiv:0910.1066] [INSPIRE].

[34] M.P. Hertzberg, M. Tegmark and F. Wilczek, Axion Cosmology and the Energy Scale of Inflation, Phys. Rev. D 78 (2008) 083507 [arXiv:0807.1726] [InSPIRE].

[35] T. Hambye, F.S. Ling, L. Lopez Honorez and J. Rocher, Scalar Multiplet Dark Matter, JHEP 07 (2009) 090 [Erratum ibid. 1005 (2010) 066] [arXiv:0903.4010] [INSPIRE].

[36] J.M. Arnold, B. Fornal and M. Trott, Prospects and Constraints for Vector-like MFV Matter at LHC, JHEP 08 (2010) 059 [arXiv: 1005.2185] [INSPIRE].

[37] Y. Grossman, Y. Nir, J. Thaler, T. Volansky and J. Zupan, Probing minimal flavor violation at the LHC, Phys. Rev. D 76 (2007) 096006 [arXiv:0706.1845] [INSPIRE].

[38] I.P. Ivanov, Minkowski space structure of the Higgs potential in 2HDM, Phys. Rev. D 75 (2007) 035001 [Erratum ibid. D 76 (2007) 039902] [hep-ph/0609018] [INSPIRE].

[39] B. Świeżewska, Yukawa independent constraints for two-Higgs-doublet models with a 125 GeV Higgs boson, Phys. Rev. D 88 (2013) 055027 [arXiv: 1209.5725] [INSPIRE].

[40] A. Ilnicka, M. Krawczyk and T. Robens, Inert Doublet Model in light of LHC Run I and astrophysical data, Phys. Rev. D 93 (2016) 055026 [arXiv: 1508.01671] [INSPIRE].

[41] P.M. Ferreira and B. Swiezewska, One-loop contributions to neutral minima in the inert doublet model, JHEP 04 (2016) 099 [arXiv: 1511. 02879] [INSPIRE].

[42] I.F. Ginzburg and I.P. Ivanov, Tree-level unitarity constraints in the most general 2HDM, Phys. Rev. D 72 (2005) 115010 [hep-ph/0508020] [INSPIRE].

[43] E. Lundstrom, M. Gustafsson and J. Edsjo, The Inert Doublet Model and LEP II Limits, Phys. Rev. D 79 (2009) 035013 [arXiv: 0810.3924] [InSPIRE].

[44] G. Bélanger, B. Dumont, U. Ellwanger, J.F. Gunion and S. Kraml, Global fit to Higgs signal strengths and couplings and implications for extended Higgs sectors, Phys. Rev. D 88 (2013) 075008 [arXiv: 1306 .2941] [INSPIRE].

[45] G. Bélanger, B. Dumont, A. Goudelis, B. Herrmann, S. Kraml and D. Sengupta, Dilepton constraints in the Inert Doublet Model from Run 1 of the LHC, Phys. Rev. D 91 (2015) 115011 [arXiv: 1503.07367] [INSPIRE]. 
[46] E. Dolle, X. Miao, S. Su and B. Thomas, Dilepton Signals in the Inert Doublet Model, Phys. Rev. D 81 (2010) 035003 [arXiv:0909.3094] [InSPIRE].

[47] S. Kraml et al., SModelS: a tool for interpreting simplified-model results from the LHC and its application to supersymmetry, Eur. Phys. J. C 74 (2014) 2868 [arXiv:1312.4175] [INSPIRE].

[48] M. Drees, H. Dreiner, D. Schmeier, J. Tattersall and J.S. Kim, CheckMATE: Confronting your Favourite New Physics Model with LHC Data, Comput. Phys. Commun. 187 (2015) 227 [arXiv: 1312.2591] [INSPIRE].

[49] J. Alwall, M. Herquet, F. Maltoni, O. Mattelaer and T. Stelzer, MadGraph 5: Going Beyond, JHEP 06 (2011) 128 [arXiv: 1106. 0522] [INSPIRE].

[50] T. Sjöstrand, S. Mrenna and P.Z. Skands, PYTHIA 6.4 Physics and Manual, JHEP 05 (2006) 026 [hep-ph/0603175] [INSPIRE].

[51] DELPHES 3 collaboration, J. de Favereau et al., DELPHES 3, a modular framework for fast simulation of a generic collider experiment, JHEP 02 (2014) 057 [arXiv:1307.6346] [INSPIRE].

[52] A. Alloul, N.D. Christensen, C. Degrande, C. Duhr and B. Fuks, FeynRules 2.0 - A complete toolbox for tree-level phenomenology, Comput. Phys. Commun. 185 (2014) 2250 [arXiv:1310.1921] [INSPIRE].

[53] M.L. Mangano, M. Moretti, F. Piccinini and M. Treccani, Matching matrix elements and shower evolution for top-quark production in hadronic collisions, JHEP 01 (2007) 013 [hep-ph/0611129] [INSPIRE].

[54] CMS collaboration, Search for dark matter, extra dimensions and unparticles in monojet events in proton-proton collisions at $\sqrt{s}=8 \mathrm{TeV}$, Eur. Phys. J. C 75 (2015) 235 [arXiv: 1408.3583] [INSPIRE].

[55] M. Kamionkowski, WIMP and axion dark matter, hep-ph/9710467 [INSPIRE].

[56] K.-Y. Choi, J.E. Kim, H.M. Lee and O. Seto, Neutralino dark matter from heavy axino decay, Phys. Rev. D 77 (2008) 123501 [arXiv:0801.0491] [INSPIRE].

[57] C. Corianò, M. Guzzi, N. Irges and A. Mariano, Axion and Neutralinos from Supersymmetric Extensions of the Standard Model with anomalous U(1)'s, Phys. Lett. B 671 (2009) 87 [arXiv:0811.0117] [inSPIRE].

[58] F.D. Steffen, Dark Matter Candidates - Axions, Neutralinos, Gravitinos and Axinos, Eur. Phys. J. C 59 (2009) 557 [arXiv:0811.3347] [InSPIRE].

[59] J. Jaeckel, The Physics Case for Axions, WIMPs, WISPs and Other Weird Stuff, in proceedings of the 4th Patras Workshop on Axions, WIMPs and WISPs (AXION-WIMP 2008), Hamburg, Germany, June 18-21, 2008, pp. 3-8, arXiv:0809.3112 [DOI].

[60] H. Baer, A. Lessa, S. Rajagopalan and W. Sreethawong, Mixed axion/neutralino cold dark matter in supersymmetric models, JCAP 06 (2011) 031 [arXiv:1103.5413] [INSPIRE].

[61] K.J. Bae, H. Baer and E.J. Chun, Mixed axion/neutralino dark matter in the SUSY DFSZ axion model, JCAP 12 (2013) 028 [arXiv:1309.5365] [INSPIRE].

[62] K.J. Bae, H. Baer, E.J. Chun and C.S. Shin, Mixed axion/gravitino dark matter from SUSY models with heavy axinos, Phys. Rev. D 91 (2015) 075011 [arXiv:1410.3857] [InSPIRE].

[63] Y.V. Stadnik and V.V. Flambaum, Nuclear spin-dependent interactions: Searches for WIMP, Axion and Topological Defect Dark Matter and Tests of Fundamental Symmetries, Eur. Phys. J. C 75 (2015) 110 [arXiv:1408.2184] [inSPIRE]. 
[64] F.S. Queiroz, K. Sinha and W. Wester, Rich tapestry: Supersymmetric axions, dark radiation and inflationary reheating, Phys. Rev. D 90 (2014) 115009 [arXiv:1407.4110] [INSPIRE].

[65] K.J. Bae, H. Baer, H. Serce and Y.-F. Zhang, Leptogenesis scenarios for natural SUSY with mixed axion-higgsino dark matter, JCAP 01 (2016) 012 [arXiv:1510.00724] [INSPIRE].

[66] K.J. Bae, H. Baer, A. Lessa and H. Serce, Mixed axion-wino dark matter, Front. Phys. 3 (2015) 49 [arXiv: 1502.07198].

[67] G. Bélanger, F. Boudjema, A. Pukhov and A. Semenov, MicrOMEGAs 2.0: A program to calculate the relic density of dark matter in a generic model, Comput. Phys. Commun. 176 (2007) 367 [hep-ph/0607059] [INSPIRE].

[68] G. Bélanger, F. Boudjema, A. Pukhov and A. Semenov, Dark matter direct detection rate in a generic model with MicrOMEGAs 2.2, Comput. Phys. Commun. 180 (2009) 747 [arXiv:0803.2360] [INSPIRE].

[69] F.S. Queiroz and C.E. Yaguna, The CTA aims at the Inert Doublet Model, JCAP 02 (2016) 038 [arXiv: 1511.05967] [INSPIRE].

[70] C. Garcia-Cely, M. Gustafsson and A. Ibarra, Probing the Inert Doublet Dark Matter Model with Cherenkov Telescopes, JCAP 02 (2016) 043 [arXiv: 1512.02801] [INSPIRE].

[71] M. Cirelli, N. Fornengo and A. Strumia, Minimal dark matter, Nucl. Phys. B 753 (2006) 178 [hep-ph/0512090] [INSPIRE].

[72] V. Silveira and A. Zee, Scalar Phantoms, Phys. Lett. B 161 (1985) 136 [INSPIRE].

[73] J. McDonald, Gauge singlet scalars as cold dark matter, Phys. Rev. D 50 (1994) 3637 [hep-ph/0702143] [INSPIRE].

[74] C.P. Burgess, M. Pospelov and T. ter Veldhuis, The minimal model of nonbaryonic dark matter: A singlet scalar, Nucl. Phys. B 619 (2001) 709 [hep-ph/0011335] [INSPIRE].

[75] D. O'Connell, M.J. Ramsey-Musolf and M.B. Wise, Minimal Extension of the Standard Model Scalar Sector, Phys. Rev. D 75 (2007) 037701 [hep-ph/0611014] [INSPIRE].

[76] V. Barger, P. Langacker, M. McCaskey, M.J. Ramsey-Musolf and G. Shaughnessy, LHC Phenomenology of an Extended Standard Model with a Real Scalar Singlet, Phys. Rev. D 77 (2008) 035005 [arXiv:0706.4311] [INSPIRE].

[77] M. Farina, D. Pappadopulo and A. Strumia, CDMS stands for Constrained Dark Matter Singlet, Phys. Lett. B 688 (2010) 329 [arXiv:0912.5038] [INSPIRE].

[78] M. Kadastik, K. Kannike, A. Racioppi and M. Raidal, Implications of the $125 \mathrm{GeV}$ Higgs boson for scalar dark matter and for the CMSSM phenomenology, JHEP 05 (2012) 061 [arXiv:1112.3647] [INSPIRE].

[79] J.K. Mizukoshi, C.A. de S. Pires, F.S. Queiroz and P.S. Rodrigues da Silva, WIMPs in a 3-3-1 model with heavy Sterile neutrinos, Phys. Rev. D 83 (2011) 065024

[arXiv: 1010.4097] [INSPIRE].

[80] J.D. Ruiz-Alvarez, C.A. de S. Pires, F.S. Queiroz, D. Restrepo and P.S. Rodrigues da Silva, On the Connection of Gamma-Rays, Dark Matter and Higgs Searches at LHC, Phys. Rev. D 86 (2012) 075011 [arXiv: 1206.5779] [INSPIRE].

[81] A. Djouadi, A. Falkowski, Y. Mambrini and J. Quevillon, Direct Detection of Higgs-Portal Dark Matter at the LHC, Eur. Phys. J. C 73 (2013) 2455 [arXiv:1205.3169] [InSPIRE].

[82] J.M. Cline, K. Kainulainen, P. Scott and C. Weniger, Update on scalar singlet dark matter, Phys. Rev. D 88 (2013) 055025 [arXiv: 1306.4710] [INSPIRE]. 
[83] A. Dasgupta and D. Borah, Scalar Dark Matter with Type II Seesaw, Nucl. Phys. B 889 (2014) 637 [arXiv: 1404.5261] [INSPIRE].

[84] D. Cogollo, A.X. Gonzalez-Morales, F.S. Queiroz and P.R. Teles, Excluding the Light Dark Matter Window of a 331 Model Using LHC and Direct Dark Matter Detection Data, JCAP 11 (2014) 002 [arXiv:1402.3271] [INSPIRE].

[85] A. Alves, S. Profumo, F.S. Queiroz and W. Shepherd, Effective field theory approach to the Galactic Center gamma-ray excess, Phys. Rev. D 90 (2014) 115003 [arXiv:1403.5027] [INSPIRE].

[86] F.S. Queiroz, K. Sinha and A. Strumia, Leptoquarks, Dark Matter and Anomalous LHC Events, Phys. Rev. D 91 (2015) 035006 [arXiv:1409.6301] [INSPIRE].

[87] L. Feng, S. Profumo and L. Ubaldi, Closing in on singlet scalar dark matter: LUX, invisible Higgs decays and gamma-ray lines, JHEP 03 (2015) 045 [arXiv:1412.1105] [INSPIRE].

[88] M. Duerr, P. Fileviez Perez and J. Smirnov, Scalar Singlet Dark Matter and Gamma Lines, Phys. Lett. B 751 (2015) 119 [arXiv: 1508.04418] [InSPIRE].

[89] M. Duerr, P. Fileviez Pérez and J. Smirnov, Scalar Dark Matter: Direct vs. Indirect Detection, JHEP 06 (2016) 152 [arXiv:1509.04282] [INSPIRE].

[90] A. Beniwal et al., Combined analysis of effective Higgs portal dark matter models, Phys. Rev. D 93 (2016) 115016 [arXiv: 1512.06458] [INSPIRE].

[91] H. Han and S. Zheng, New Constraints on Higgs-portal Scalar Dark Matter, JHEP 12 (2015) 044 [arXiv: 1509.01765] [InSPIRE].

[92] G. Dupuis, Collider Constraints and Prospects of a Scalar Singlet Extension to Higgs Portal Dark Matter, JHEP 07 (2016) 008 [arXiv: 1604.04552] [INSPIRE].

[93] H. Han, J.M. Yang, Y. Zhang and S. Zheng, Collider Signatures of Higgs-portal Scalar Dark Matter, Phys. Lett. B 756 (2016) 109 [arXiv: 1601.06232] [INSPIRE].

[94] N. Blinov, J. Kozaczuk, D.E. Morrissey and A. de la Puente, Compressing the Inert Doublet Model, Phys. Rev. D 93 (2016) 035020 [arXiv: 1510.08069] [INSPIRE].

[95] G. Bélanger, F. Boudjema, A. Pukhov and A. Semenov, MicrOMEGAs 3 : A program for calculating dark matter observables, Comput. Phys. Commun. 185 (2014) 960 [arXiv: 1305.0237] [INSPIRE].

[96] D. Tucker-Smith and N. Weiner, Inelastic dark matter, Phys. Rev. D 64 (2001) 043502 [hep-ph/0101138] [INSPIRE].

[97] C. Arina, F.-S. Ling and M.H.G. Tytgat, IDM and iDM or The Inert Doublet Model and Inelastic Dark Matter, JCAP 10 (2009) 018 [arXiv:0907.0430] [INSPIRE].

[98] WMAP collaboration, E. Komatsu et al., Seven-Year Wilkinson Microwave Anisotropy Probe (WMAP) Observations: Cosmological Interpretation, Astrophys. J. Suppl. 192 (2011) 18 [arXiv: 1001.4538] [INSPIRE].

[99] LUX collaboration, D.S. Akerib et al., Improved Limits on Scattering of Weakly Interacting Massive Particles from Reanalysis of 2013 LUX Data, Phys. Rev. Lett. 116 (2016) 161301 [arXiv: 1512.03506] [INSPIRE].

[100] SuperCDMS collaboration, R. Agnese et al., Search for Low-Mass Weakly Interacting Massive Particles with SuperCDMS, Phys. Rev. Lett. 112 (2014) 241302 [arXiv: 1402.7137] [INSPIRE].

[101] PICO collaboration, C. Amole et al., Dark Matter Search Results from the PICO-2L $C_{3} F_{8}$ Bubble Chamber, Phys. Rev. Lett. 114 (2015) 231302 [arXiv:1503.00008] [INSPIRE]. 
[102] DarkSide collaboration, P. Agnes et al., First Results from the DarkSide-50 Dark Matter Experiment at Laboratori Nazionali del Gran Sasso, Phys. Lett. B 743 (2015) 456 [arXiv: 1410.0653] [INSPIRE].

[103] PandaX collaboration, M. Xiao et al., First dark matter search results from the PandaX-I experiment, Sci. China Phys. Mech. Astron. 57 (2014) 2024 [arXiv:1408.5114] [InSPIRE].

[104] PICASSO collaboration, S. Archambault et al., Constraints on Low-Mass WIMP Interactions on ${ }^{19} F$ from PICASSO, Phys. Lett. B 711 (2012) 153 [arXiv:1202.1240] [INSPIRE].

[105] IcECuBE collaboration, M.G. Aartsen et al., Improved limits on dark matter annihilation in the Sun with the 79-string IceCube detector and implications for supersymmetry, JCAP 04 (2016) 022 [arXiv: 1601.00653] [INSPIRE].

[106] Super-Kamiokande collaboration, K. Choi et al., Search for neutrinos from annihilation of captured low-mass dark matter particles in the Sun by Super-Kamiokande, Phys. Rev. Lett. 114 (2015) 141301 [arXiv: 1503.04858] [INSPIRE].

[107] Super-Kamiokande collaboration, P. Mijakowski, Indirect searches for dark matter particles at Super-Kamiokande, J. Phys. Conf. Ser. 718 (2016) 042040 [INSPIRE].

[108] XENON1T collaboration, E. Aprile, The XENON1T Dark Matter Search Experiment, Springer Proc. Phys. 148 (2013) 93 [arXiv: 1206.6288].

[109] XENON collaboration, E. Aprile et al., Physics reach of the XENON1T dark matter experiment, JCAP 04 (2016) 027 [arXiv: 1512.07501] [INSPIRE].

[110] F. Giacchino, A. Ibarra, L. Lopez Honorez, M.H.G. Tytgat and S. Wild, Signatures from Scalar Dark Matter with a Vector-like Quark Mediator, JCAP 02 (2016) 002 [arXiv: 1511.04452] [INSPIRE].

[111] S. Profumo, Astrophysical Probes of Dark Matter, arXiv:1301.0952 [INSPIRE].

[112] F.S. Queiroz, Dark Matter Overview: Collider, Direct and Indirect Detection Searches, arXiv: 1605.08788 [INSPIRE].

[113] G. Bertone and D. Hooper, A History of Dark Matter, Submitted to: Rev. Mod. Phys. (2016) [arXiv: 1605.04909] [INSPIRE].

[114] D. Hooper, C. Kelso and F.S. Queiroz, Stringent and Robust Constraints on the Dark Matter Annihilation Cross section From the Region of the Galactic Center, Astropart. Phys. 46 (2013) 55 [arXiv:1209.3015] [INSPIRE].

[115] L. Bergstrom, T. Bringmann, I. Cholis, D. Hooper and C. Weniger, New limits on dark matter annihilation from AMS cosmic ray positron data, Phys. Rev. Lett. 111 (2013) 171101 [arXiv: 1306.3983] [INSPIRE].

[116] A. Berlin and D. Hooper, Stringent Constraints on the Dark Matter Annihilation Cross section From Subhalo Searches with the Fermi Gamma-Ray Space Telescope, Phys. Rev. D 89 (2014) 016014 [arXiv:1309.0525] [INSPIRE].

[117] A.X. Gonzalez-Morales, S. Profumo and F.S. Queiroz, Effect of Black Holes in Local Dwarf Spheroidal Galaxies on Gamma-Ray Constraints on Dark Matter Annihilation, Phys. Rev. D 90 (2014) 103508 [arXiv:1406.2424] [InSPIRE].

[118] B. Dutta, Y. Gao, T. Ghosh and L.E. Strigari, Confronting Galactic center and dwarf spheroidal gamma-ray observations with cascade annihilation models, Phys. Rev. D 92 (2015) 075019 [arXiv: 1508.05989] [INSPIRE]. 
[119] K.K. Boddy and J. Kumar, Indirect Detection of Dark Matter Using MeV-Range Gamma-Ray Telescopes, Phys. Rev. D 92 (2015) 023533 [arXiv: 1504.04024] [INSPIRE].

[120] A.A. Kaurov, D. Hooper and N.Y. Gnedin, The Effects of Dark Matter Annihilation on Cosmic Reionization, Submitted to: Astrophys. J. (2015) [arXiv:1512.00526] [INSPIRE].

[121] T. Bringmann, A.J. Galea and P. Walia, Leading QCD Corrections for Indirect Dark Matter Searches: a Fresh Look, Phys. Rev. D 93 (2016) 043529 [arXiv: 1510. 02473] [InSPIRE].

[122] J. Kumar, J. Liao and D. Marfatia, Dark matter annihilation with s-channel internal Higgsstrahlung, Phys. Lett. B 759 (2016) 277 [arXiv:1605.00611] [INSPIRE].

[123] F.S. Queiroz, C.E. Yaguna and C. Weniger, Gamma-ray Limits on Neutrino Lines, JCAP 05 (2016) 050 [arXiv: 1602.05966] [INSPIRE].

[124] M.G. Baring, T. Ghosh, F.S. Queiroz and K. Sinha, New Limits on the Dark Matter Lifetime from Dwarf Spheroidal Galaxies using Fermi-LAT, Phys. Rev. D 93 (2016) 103009 [arXiv: 1510.00389] [INSPIRE].

[125] S. Profumo, F.S. Queiroz and C.E. Yaguna, Extending Fermi-LAT and H.E.S.S. Limits on Gamma-ray Lines from Dark Matter Annihilation, arXiv:1602.08501 [INSPIRE].

[126] H.E.S.S. collaboration, A. Abramowski et al., Search for a Dark Matter annihilation signal from the Galactic Center halo with H.E.S.S, Phys. Rev. Lett. 106 (2011) 161301 [arXiv:1103.3266] [INSPIRE].

[127] V. Lefranc, E. Moulin, P. Panci and J. Silk, Prospects for Annihilating Dark Matter in the inner Galactic halo by the Cherenkov Telescope Array, Phys. Rev. D 91 (2015) 122003 [arXiv: 1502.05064] [INSPIRE].

[128] H. Silverwood, C. Weniger, P. Scott and G. Bertone, A realistic assessment of the CTA sensitivity to dark matter annihilation, JCAP 03 (2015) 055 [arXiv: 1408.4131] [INSPIRE].

[129] ADMX collaboration, S.J. Asztalos et al., Design and performance of the ADMX SQUID-based microwave receiver, Nucl. Instrum. Meth. A 656 (2011) 39 [arXiv:1105.4203] [INSPIRE].

[130] E. Armengaud et al., Conceptual Design of the International Axion Observatory (IAXO), 2014 JINST 9 T05002 [arXiv: 1401.3233] [INSPIRE].

[131] D.C. Malling et al., After LUX: The LZ Program, arXiv:1110.0103 [InSPIRE].

[132] V. Barger, L.L. Everett, H.E. Logan and G. Shaughnessy, Scrutinizing the $125 \mathrm{GeV}$ Higgs boson in two Higgs doublet models at the LHC, ILC and Muon Collider, Phys. Rev. D 88 (2013) 115003 [arXiv:1308.0052] [INSPIRE].

[133] L. Wang and X.-F. Han, Study of the heavy CP-even Higgs with mass $125 \mathrm{GeV}$ in two-Higgs-doublet models at the LHC and ILC, JHEP 11 (2014) 085 [arXiv:1404.7437] [INSPIRE].

[134] J. Hajer, Y.-Y. Li, T. Liu and J.F.H. Shiu, Heavy Higgs Bosons at 14 TeV and $100 \mathrm{TeV}$, JHEP 11 (2015) 124 [arXiv:1504.07617] [INSPIRE].

[135] N. Arkani-Hamed, T. Han, M. Mangano and L.-T. Wang, Physics Opportunities of a $100 \mathrm{TeV}$ Proton-Proton Collider, Phys. Rept. 652 (2016) 1 [arXiv:1511.06495] [InSPIRE].

[136] T. Golling et al., Physics at a $100 \mathrm{TeV}$ pp collider: beyond the Standard Model phenomena, arXiv: 1606.00947 [INSPIRE]. 